\title{
Pacific
}

Journal of

Mathematics

\section{GRAPHS OF BOUNDED DEGREE} AND THE $p$-HARMONIC BOUNDARY

\author{
Michael J. Puls
}




\title{
GRAPHS OF BOUNDED DEGREE AND THE $p$-HARMONIC BOUNDARY
}

\author{
Michael J. PUlS
}

\begin{abstract}
Let $p$ be a real number greater than one and let $G$ be a connected graph of bounded degree. We introduce the $p$-harmonic boundary of $G$ and use it to characterize the graphs $G$ for which the constant functions are the only $p$ harmonic functions on $G$. We show that any continuous function on the $p$ harmonic boundary of $G$ can be extended to a function that is $p$-harmonic on $G$. We also give some properties of this boundary that are preserved under rough-isometries. Now let $\Gamma$ be a finitely generated group. As an application of our results, we characterize the vanishing of the first reduced $\ell^{p}$-cohomology of $\Gamma$ in terms of the cardinality of its $p$-harmonic boundary. We also study the relationship between translation invariant linear functionals on a certain difference space of functions on $\Gamma$, the $p$-harmonic boundary of $\Gamma$, and the first reduced $\ell^{p}$-cohomology of $\Gamma$.
\end{abstract}

\section{Introduction}

Let $p$ be a real number greater than one and let $\Gamma$ be a finitely generated infinite group. There has been some work done relating various boundaries of $\Gamma$ and the nonvanishing of the first reduced $\ell^{p}$-cohomology space $\bar{H}_{(p)}^{1}(\Gamma)$ of $\Gamma$ (to be defined in Section 7). Gromov [1993, Chapter 8, Section C2] - see also [Elek 1997] - showed that if the $\ell^{p}$-corona of $\Gamma$ contains more than one element, then $\bar{H}_{(p)}^{1}(\Gamma) \neq 0$. Puls [2007] showed that if there is a Floyd boundary of $\Gamma$ containing more than two elements, and if the Floyd admissible function satisfies a certain decay condition, then $\bar{H}_{(p)}^{1}(\Gamma) \neq 0$. However, it is unknown if the converse of either of these two results is true. The motivation for this paper is to find a boundary for $\Gamma$ whose cardinality characterizes the vanishing of $\bar{H}_{(p)}^{1}(\Gamma)$. We will show that the $p$-harmonic boundary, defined in Section 2.1, does the trick. This boundary gives the desired result because $\bar{H}_{(p)}^{1}(\Gamma)=0$ if and only if the only $p$-harmonic functions on $\Gamma$ are the constants, [Puls 2006, Theorem 3.5]. We will show in Section 7 that the cardinality of the $p$-harmonic boundary is 0 or 1 if and

MSC2000: primary 60J50; secondary 43A15, 31C20.

Keywords: Royden boundary, $p$-harmonic boundary, $p$-harmonic function, rough isometry,

$\ell^{p}$-cohomology, translation invariant functionals.

The research for this paper was partially supported by PSC-CUNY grant 60123-38 39 . 
only if the only $p$-harmonic functions on $\Gamma$ are the constants. Hence, $\bar{H}_{(p)}^{1}(\Gamma)=0$ if and only if the cardinality of the $p$-harmonic boundary is 0 or 1 .

$L_{p}$-cohomology was investigated first in [Gol'dshteřn et al. 1987] for the case of Riemannian manifolds. Gromov [1993, Chapter 8] has studied $\ell^{p}$-cohomology for finitely generated groups, and in the more general setting of graphs with bounded degree. In particular, Cheeger and Gromov [1986] showed that the first reduced $\ell^{2}$-cohomology of a finitely generated amenable group is zero. Gromov [1993, page 150] conjectured that this result is also true for all real numbers $p>1$. This is our main justification for choosing to study the $p$-harmonic boundary in the discrete setting. If enough insight can be gained into this boundary, then we may be able to develop the tools needed to compute the $p$-harmonic boundary of a finitely generated amenable group. This of course would resolve Gromov's conjecture.

More information about the first reduced $L_{p}$-cohomology (and the special case of $L_{2}$-cohomology) can be found in [Pansu 1989; 2007; 2008; Tessera 2009] for various manifolds, and in [Bekka and Valette 1997; Bourdon 2004; Bourdon et al. 2005; Elek 1998; Martin and Valette 2007; Puls 2003; 2006; 2007] for finitely generated groups. As implied earlier, there is a strong connection between the vanishing of the first reduced $L_{p}$-cohomology and the nonexistence nonconstant $p$-harmonic functions; for a proof in the case of homogeneous Riemannian manifolds, see [Tessera 2009, Proposition 4.11]. Thus results on $p$-harmonic functions are useful in trying to determine if the first reduced $L_{p}$-cohomology vanishes. The papers [Coulhon et al. 2001; Grigoryan 1987] study p-harmonic functions on manifolds, while [Holopainen and Soardi 1997a; Kim and Lee 2005; 2007; Soardi 1993; Yamasaki 1977] examine $p$-harmonic functions on graphs.

\section{Definitions and statement of main results}

Let $p$ be a real number greater than one, and let $\Gamma$ be a finitely generated infinite group. The definition of the $p$-harmonic boundary for $\Gamma$ does not depend on the group law of $\Gamma$, so we can define this boundary in the more general setting of a graph. The reason is that we can associate a graph, called the Cayley graph of $\Gamma$, with $\Gamma$. The vertex set for this graph consists of the elements of $\Gamma$, and $x_{1}, x_{2} \in \Gamma$ are joined by an edge if and only if $x_{1}=x_{2} s^{ \pm 1}$ for a generator $s$ of $\Gamma$.

2.1. The p-harmonic boundary. Let $G$ be a graph with vertex set $V_{G}$ and edge set $E_{G}$. We will write $V$ for $V_{G}$ and $E$ for $E_{G}$ if it is clear what the graph $G$ is. For $x \in V$, we denote by $\operatorname{deg}(x)$ the number of neighbors of $x$ and by $N_{x}$ the set of neighbors of $x$. We say a graph $G$ is of bounded degree if there exists a positive integer $k$ such that $\operatorname{deg}(x) \leq k$ for every $x \in V$. A path in $G$ is a sequence of vertices $x_{1}, x_{2}, \ldots, x_{n}$ for which $x_{i+1} \in N_{x_{i}}$ for $1 \leq i \leq n-1$. A graph $G$ is connected if any two given vertices of $G$ are joined by a path. All graphs considered in this 
paper will be countably infinite, connected, of bounded degree with no self-loops. Assign length one to each edge in $E_{G}$; then the graph $G$ is a metric space with respect to the shortest path metric. Let $d_{G}(\cdot, \cdot)$ denote this metric. So if $x, y \in V$, then $d_{G}(x, y)$ is the length of the shortest path joining $x$ and $y$. We will drop the subscript $G$ from $d_{G}(\cdot, \cdot)$ when it is clear what graph $G$ we are working with. Finally, if $x \in V$, then $B_{n}(x)$ will denote the metric ball that contains all elements of $V$ that have distance less than $n$ from $x$.

Let $G$ be a graph with vertex set $V$, and let $p$ be a real number greater than one. To construct the $p$-harmonic boundary of $G$, we need to first define the space of bounded $p$-Dirichlet finite functions on $G$. For any $S \subset V$, the outer boundary $\partial S$ of $S$ is the set of vertices in $V \backslash S$ with at least one neighbor in $S$. For a real-valued function $f$ on $S \cup \partial S$, we define the $p$-th power of the gradient, the $p$-Dirichlet sum, and the $p$-Laplacian of $x \in S$ by

$$
\begin{aligned}
|D f(x)|^{p} & =\sum_{y \in N_{x}}|f(y)-f(x)|^{p}, \\
I_{p}(f, S) & =\sum_{x \in S}|D f(x)|^{p}, \\
\Delta_{p} f(x) & =\sum_{y \in N_{x}}|f(y)-f(x)|^{p-2}(f(y)-f(x)) .
\end{aligned}
$$

In the case $1<p<2$, we make the convention that

$$
|f(y)-f(x)|^{p-2}(f(y)-f(x))=0 \quad \text { if } f(y)=f(x) .
$$

Let $S \subseteq V$. We say a function $f$ is $p$-harmonic on $S$ if $\Delta_{p} f(x)=0$ for all $x \in S$, and $p$-Dirichlet finite if $I_{p}(f, V)<\infty$. We denote the set of all $p$-Dirichlet finite functions on $G$ by $D_{p}(G)$. Under the norm

$$
\|f\|_{D_{p}}=\left(I_{p}(f, V)+|f(o)|^{p}\right)^{1 / p},
$$

$D_{p}(G)$ is a reflexive Banach space, where $o$ is a fixed vertex of $G$ and $f \in D_{p}(G)$. Denote by $\operatorname{HD}_{p}(G)$ the set of $p$-harmonic functions on $V$ contained in $D_{p}(G)$. Let $\ell^{\infty}(G)$ denote the set of bounded functions on $V$, and let $\|f\|_{\infty}=\sup _{V}|f|$ for $f \in \ell^{\infty}(G)$. Set $\mathrm{BD}_{p}(G)=D_{p}(G) \cap \ell^{\infty}(G)$. The set $\mathrm{BD}_{p}(G)$ is a Banach space under the norm

$$
\|f\|_{\mathrm{BD}_{p}}=\left(I_{p}(f, V)\right)^{1 / p}+\|f\|_{\infty},
$$

where $f \in \mathrm{BD}_{p}(G)$. Set $\mathrm{BHD}_{p}(G)=\operatorname{HD}_{p}(G) \cap \mathrm{BD}_{p}(G)$. It turns out that $\mathrm{BD}_{p}(G)$ is closed under pointwise multiplication. To see this, let $f, h \in \operatorname{BD}_{p}(G)$ and set $a=\sup _{V}|f|$ and $b=\sup _{V}|h|$. It follows from Minkowski's inequality that

$$
\left(I_{p}(f h, V)\right)^{1 / p} \leq b\left(I_{p}(f, V)\right)^{1 / p}+a\left(I_{p}(h, V)\right)^{1 / p} .
$$


Thus $f h \in \mathrm{BD}_{p}(G)$. Using the inequality above, we obtain

$$
\|f h\|_{\mathrm{BD}_{p}} \leq\left(\left(I_{p}(f, V)\right)^{1 / p}+a\right)\left(\left(I_{p}(h, V)\right)^{1 / p}+b\right)=\|f\|_{\mathrm{BD}_{p}}\|h\|_{\mathrm{BD}_{p}} .
$$

Hence $\mathrm{BD}_{p}(G)$ is an abelian Banach algebra. A character on $\mathrm{BD}_{p}(G)$ is a nonzero homomorphism from $\operatorname{BD}_{p}(G)$ into the complex numbers. Let $\operatorname{Sp}\left(\operatorname{BD}_{p}(G)\right)$ be the set of characters on $\operatorname{BD}_{p}(G)$; it is known as the spectrum of $\operatorname{BD}_{p}(G)$. With respect to the weak $*$-topology, $\operatorname{Sp}\left(\mathrm{BD}_{p}(G)\right)$ is a compact Hausdorff space. Let $C\left(\operatorname{Sp}\left(\operatorname{BD}_{p}(G)\right)\right)$ denote the set of continuous functions on $\operatorname{Sp}\left(\operatorname{BD}_{p}(G)\right)$. For each $f \in \mathrm{BD}_{p}(G)$, we define a continuous function $\hat{f}$ on $\operatorname{Sp}\left(\mathrm{BD}_{p}(G)\right)$ by $\hat{f}(\tau)=\tau(f)$. The map $f \rightarrow \hat{f}$ is known as the Gelfand transform.

Define a map $i: V \rightarrow \operatorname{Sp}\left(\operatorname{BD}_{p}(G)\right)$ by $(i(x))(f)=f(x)$. For $x \in V$, define $\delta_{x}$ by $\delta_{x}(v)=0$ if $v \neq x$ and $\delta_{x}(x)=1$. Let $x, y \in V$ and suppose $i(x)=i(y)$; then $(i(x))\left(\delta_{x}\right)=(i(y))\left(\delta_{x}\right)$, which implies $\delta_{x}(x)=\delta_{x}(y)$. Thus $i$ is an injection. If $f$ is a nonzero function in $\operatorname{BD}_{p}(G)$, then there exists an $x \in V$ such that $\hat{f}(i(x)) \neq 0$ since $\hat{f}(i(x))=f(x)$. Hence $\mathrm{BD}_{p}(G)$ is semisimple. Then [Taylor and Lay 1986, Theorem 4.6 on page 408] tells us that $\operatorname{BD}_{p}(G)$ is isomorphic to a subalgebra of $C\left(\operatorname{Sp}\left(\mathrm{BD}_{p}(G)\right)\right)$ via the Gelfand transform. Since the Gelfand transform separates points of $\operatorname{Sp}\left(\mathrm{BD}_{p}(G)\right)$ and the constant functions are contained in $\operatorname{BD}_{p}(G)$, the Stone-Weierstrass theorem yields that $\mathrm{BD}_{p}(G)$ is dense in $C\left(\operatorname{Sp}\left(\operatorname{BD}_{p}(G)\right)\right)$ with respect to the supremum norm. The following proposition shows that $i(V)$ is dense in $\operatorname{Sp}\left(\mathrm{BD}_{p}(G)\right)$; see [Elek 1997, Proposition 1.1(ii)] for the proof.

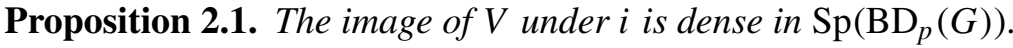

When the context is clear we will abuse notation and write $V$ for $i(V)$ and $x$ for $i(x)$, where $x \in V$. The compact Hausdorff space $\operatorname{Sp}\left(\operatorname{BD}_{p}(G)\right) \backslash V$ is known as the $p$-Royden boundary of $G$, which we will denote by $R_{p}(G)$. When $p=2$, this is simply known as the Royden boundary of $G$. Let $\mathbb{R} G$ be the set of realvalued functions on $V$ with finite support, and let $B(\overline{\mathbb{R} G})_{D_{p}}=(\overline{\mathbb{R} G})_{D_{p}} \cap \ell^{\infty}(G)$. Suppose $\left(f_{n}\right)$ is a sequence in $B(\overline{\mathbb{R} G})_{D_{p}}$ that converges to a bounded function $f$ in the $\mathrm{BD}_{p}(G)$ norm. It follows from $\left\|f-f_{n}\right\|_{D_{p}} \leq\left\|f-f_{n}\right\|_{\mathrm{BD}_{p}}$ that $f \in(\overline{\mathbb{R} G})_{D_{p}}$. Thus $B(\overline{\mathbb{R} G})_{D_{p}}$ is closed in $\mathrm{BD}_{p}(G)$ with respect to the $\mathrm{BD}_{p}(G)$ norm. We are now ready to define the main object of study for this paper.

The $p$-harmonic boundary of $G$ is the subset

$$
\partial_{p}(G):=\left\{x \in R_{p}(G) \mid \hat{f}(x)=0 \text { for all } f \in B(\overline{\mathbb{R} G})_{D_{p}}\right\}
$$

of the $p$-Royden boundary. When $p=2$, the $p$-harmonic boundary is known as the harmonic boundary. Our definition of $p$-harmonic boundary directly generalizes that of harmonic boundary. A good reference for the Royden and harmonic boundaries of graphs is [Soardi 1994, Chapter VI]. 
An important fact about $B(\overline{\mathbb{R} G})_{D_{p}}$ is that it is an ideal in $\operatorname{BD}_{p}(G)$. To see this, let $f \in B(\overline{\mathbb{R} G})_{D_{p}}$ and $h \in \mathrm{BD}_{p}(G)$. We need to show that $f h \in B(\overline{\mathbb{R} G})_{D_{p}}$. We claim that there exists a sequence $\left(f_{n}\right)$ in $\mathbb{R} G$ converging pointwise to $f$, for which there exists a constant $M$ with $\left|f_{n}(x)\right| \leq M$ for all $n$ and for all $x \in V$, and for which $I_{p}\left(f_{n}, V\right)$ is bounded. Let $\left(u_{n}\right)$ be a sequence in $\mathbb{R} G$ that converges to $f$ in $D_{p}(G)$ and let $M=\sup _{x \in V}|f(x)|$. Set $f_{n}=\max \left(\min \left(u_{n}, M\right),-M\right)$. The sequence $\left(f_{n}\right)$ satisfies the claim above since $I_{p}\left(u_{n}, V\right)$ is bounded and $I_{p}\left(f_{n}, V\right) \leq I_{p}\left(u_{n}, V\right)$. Also $\left(f_{n} h\right)$ is a sequence in $\mathbb{R} G$ that converges pointwise to $f h$. By $(2-1)$, we see that

$$
I_{p}\left(f_{n} h, V\right) \leq\left(b\left(I_{p}\left(f_{n}, V\right)\right)^{1 / p}+M\left(I_{p}(h, V)\right)^{1 / p}\right)^{p},
$$

where $b=\sup _{x \in V}|h(x)|$. Since $I_{p}\left(f_{n} h, V\right)$ is bounded, [Taylor and Lay 1986, Theorem 10.6, page 177] says, by passing to a subsequence if necessary, that $\left(f_{n} h\right)$ converges weakly to a function $\overline{f h}$. Since $B(\overline{\mathbb{R} G})_{D_{p}}$ is closed, it follows that $\overline{f h} \in B(\overline{\mathbb{R} G})_{D_{p}}$. Because point evaluations by elements of $V$ are continuous linear functionals on $\mathrm{BD}_{p}(G),\left(f_{n} h\right)$ also converges pointwise to $\overline{f h}$. Hence, $\overline{f h}=f h$ and $f h \in B(\overline{\mathbb{R} G})_{D_{p}}$.

2.2. Statement of main results. Recall that $p$ is a real number greater than one and that $o$ is a fixed vertex of $V$. By \#(A), we mean the cardinality of a set $A$, and $1_{V}$ will denote the function on $V$ that always takes the value one. Furthermore, $\ell^{p}(G)$ will be the set that consists of the functions on $V$ for which $\sum_{x \in V}|f(x)|^{p}<\infty$. The $\ell^{p}$-norm for $f \in \ell^{p}(G)$ is given by $\|f\|_{p}=\left(\sum_{x \in V}|f(x)|^{p}\right)^{1 / p}$. In Section 3, we give a quick review of some results about $p$-harmonic functions on graphs. In Section 4 we prove several results concerning $\operatorname{BD}_{p}(G)$ and $\partial_{p}(G)$; when $\operatorname{BHD}_{p}(G)$ consists precisely of the constant functions and a neighborhood base is given for the topology on $\partial_{p}(G)$, we characterize when $\partial_{p}(G)=\varnothing$,

Before we stating some of our main results, we need a theorem that will allow us to classify graphs in a nice way. We start by giving the following definition. The $p$-capacity of a finite subset $A$ of $V$ is defined by

$$
\operatorname{Cap}_{p}(A, \infty, V)=\inf _{u} I_{p}(u, V),
$$

where the infimum is taken over all finitely supported functions $u$ on $V$ such that $u=1$ on $A$. The following theorem will allow us to classify a graph $G$ in terms of the $p$-capacity of a finite set.

Theorem 2.2 [Yamasaki 1977, Theorem 3.1]. Let A be a finite, nonempty subset of $V$. Then

$$
\operatorname{Cap}_{p}(A, \infty, V)=0 \quad \text { if and only if } \quad 1_{V} \in B(\overline{\mathbb{R} G})_{D_{p}} .
$$

Corollary 2.3. Let $A$ and $B$ be nonempty finite subsets of $V$. Then

$$
\operatorname{Cap}_{p}(A, \infty, V)=0 \quad \text { if and only if } \operatorname{Cap}_{p}(B, \infty, V)=0 \text {. }
$$


We say that a graph $G$ is $p$-parabolic if there exists a finite subset $A$ of $V$ such that $\operatorname{Cap}_{p}(A, \infty, V)=0$. If $G$ is not $p$-parabolic, we shall say that $G$ is $p$-hyperbolic. If $G$ is $p$-hyperbolic, then $\operatorname{Cap}_{p}(A, \infty, V)>0$ for all finite subsets $A$ of $V$.

In Section 5 we will prove the following results. The first reduces to [Soardi 1994, Theorem 4.6] in the case $p=2$ and also generalizes [Kim and Lee 2005, Theorem 4.2].

Theorem 2.4. Let $p$ be a real number greater than one, and let $G$ be a graph. If $G$ is p-parabolic, then all p-harmonic functions on $G$ are constant functions.

Identify the constant functions on $V$ with $\mathbb{R}$. By combining this theorem with [Holopainen and Soardi 1997a, Lemma 4.4] and Theorem 4.10 we get a Liouvilletype theorem for $p$-harmonic functions:

Theorem 2.5. Let $p$ be a real number greater than one. Then $\operatorname{HD}_{p}(G)=\mathbb{R}$ if and only if the cardinality of $\partial_{p}(G)$ is either zero or one.

Theorem 2.6. Let $p$ be a real number greater than one and let $G$ be a graph. If $f$ is a continuous function on $\partial_{p}(G)$, then there exists a p-harmonic function $h$ on $V$ such that $\lim _{n \rightarrow \infty} h\left(x_{n}\right)=f(x)$, where $x \in \partial_{p}(G)$ and $\left(x_{n}\right)$ is any sequence in $V$ that converges to $x$.

By combining this theorem with the maximum principle and Corollary 4.9 we obtain the following corollary, which generalizes both [Kim and Lee 2005, Theorem 4.3] and [Kim and Lee 2007, Theorem 1.1].

Corollary 2.7. Let $p$ be a real number greater than one and let $G$ be a graph. Assume that the p-harmonic boundary of $G$ is a finite set $\left\{x_{1}, x_{2}, \ldots, x_{n}\right\}$ of points. Then given real numbers $a_{1}, a_{2}, \ldots, a_{n} \in \mathbb{R}$, there exists a bounded $p$-harmonic function $h$ that satisfies

$$
h\left(x_{i}\right)=a_{i} \quad \text { for } i=1,2, \ldots, n .
$$

Conversely, each bounded p-harmonic function is uniquely determined by its values in (2-2).

Let $\left(X, d_{X}\right)$ and $\left(Y, d_{Y}\right)$ be metric spaces. A map $\phi: X \rightarrow Y$ is said to be a rough isometry if it satisfies the following two conditions:

(1) There exist constants $a \geq 1$ and $b \geq 0$ such that for $x_{1}, x_{2} \in X$

$$
(1 / a) d_{X}\left(x_{1}, x_{2}\right)-b \leq d_{Y}\left(\phi\left(x_{1}\right), \phi\left(x_{2}\right)\right) \leq a d_{X}\left(x_{1}, x_{2}\right)+b .
$$

(2) There exists a positive constant $c$ such that for each $y \in Y$, there exists an $x \in X$ that satisfies $d_{Y}(\phi(x), y)<c$. 
For a rough isometry $\phi$, there exists a rough isometry $\psi: Y \rightarrow X$ such that if $x \in X$ and $y \in Y$, then $d_{X}((\psi \circ \phi)(x), x) \leq a(c+b)$ and $d_{Y}((\phi \circ \psi)(y), y) \leq c$. The map $\psi$, which is not unique, is said to be a rough inverse for $\phi$. Whenever we refer to a rough inverse to a rough isometry, it will always satisfy the conditions above. In Section 6, we prove the following two results:

Theorem 2.8. Let $p$ be a real number greater than one and let $G$ and $H$ be graphs. If there is a rough isometry from $G$ to $H$, then $\partial_{p}(G)$ is homeomorphic to $\partial_{p}(H)$.

Theorem 2.9. Let $p$ be a real number greater than one and let $G$ and $H$ be graphs. If there is a rough isometry from $G$ to $H$, then there is a bijection from $\operatorname{BHD}_{p}(G)$ to $\operatorname{BHD}_{p}(H)$.

The main result of [Soardi 1993] is that if $G$ and $H$ are roughly isometric graphs, then $\operatorname{HD}_{p}(G)=\mathbb{R}$ if and only if $\operatorname{HD}_{p}(H)=\mathbb{R}$. By [Holopainen and Soardi 1997a, Lemma 4.4], this is equivalent to $\operatorname{BHD}_{p}(G)=\mathbb{R}$ if and only if $\operatorname{BHD}_{p}(H)=\mathbb{R}$. Both Theorem 2.8 and Theorem 2.9 are generalizations of this result.

We now return to the case of a finitely generated group $\Gamma$. In Section 7 , we define the first reduced $\ell^{p}$-cohomology space $\bar{H}_{(p)}^{1}(\Gamma)$ of $\Gamma$. Then we will use our results on $p$-harmonic boundaries to prove this:

Theorem 2.10. Let $1<p \in \mathbb{R}$. Then $\bar{H}_{(p)}^{1}(\Gamma) \neq 0$ if and only if $\#\left(\partial_{p}(\Gamma)\right)>1$.

It appears there are not many explicit examples of the $p$-Royden boundary $R_{p}(G)$ for a given graph $G$. Wysoczański [1996] provided the only example we know of by giving an explicit description of $R_{2}(\mathbb{Z})$. We will conclude Section 7 by using Theorem 2.10 to compute the $p$-harmonic boundary for the case $\Gamma=\mathbb{Z}^{n}$. We will also compute the $p$-Royden boundary of nonamenable groups with infinite center, and of the groups $\Gamma_{1} \times \Gamma_{2} \times \cdots \times \Gamma_{n}$ for $n \geq 2$, where each $F_{i}$ is finitely generated and at least one of the $\Gamma_{i}$ is nonamenable.

Let $E$ be a normed space of functions on a finitely generated group $\Gamma$. Let $f \in E$ and let $x \in \Gamma$. The right translation of $f$ by $x$, denoted by $f_{x}$, is the function $f_{x}(g)=f\left(g x^{-1}\right)$, where $g \in \Gamma$. Assume that if $f \in E$, then $f_{x} \in E$ for all $x \in \Gamma$, that is, that $E$ is right translation invariant. For the rest of this paper translation invariant will mean right translation invariant. We shall say that $T$ is a translation invariant linear functional (TILF) on $E$ if $T\left(f_{x}\right)=T(f)$ for $f \in E$ and $x \in \Gamma$. We will use TILFs to denote translation invariant linear functionals. A common question to ask is, If $T$ is a TILF on $E$, then is $T$ continuous? For background about the problem of automatic continuity, see [Meisters 1983; Saeki 1984; Willis 1988; Woodward 1974]. Define

$$
\operatorname{Diff}(E):=\text { linear } \operatorname{span}\left\{f_{x}-f \mid f \in E, x \in \Gamma\right\} .
$$

It is clear that $\operatorname{Diff}(E)$ is contained in the kernel of any TILF on $E$. In Section 8 we study TILFs on $D_{p}(\Gamma) / \mathbb{R}$, and prove the following: 
Theorem 2.11. Let $\Gamma$ be a finitely generated infinite group and let $1<p \in \mathbb{R}$. Then $\#\left(\partial_{p}(\Gamma)\right)>1$ if and only if there exists a nonzero continuous TILF on $D_{p}(\Gamma) / \mathbb{R}$.

Willis [1986] showed that if $\Gamma$ is nonamenable, then the only TILF on $\ell^{p}(\Gamma)$ is the zero functional. (Consequently every TILF is automatically continuous!) We will conclude Section 8 by showing that this result is not true for $D_{p}(\Gamma) / \mathbb{R}$.

\section{Review of $p$-harmonic functions on graphs}

The four results below are from [Holopainen and Soardi 1997a, Section 3], where a more comprehensive treatment, including proofs, is given.

- Existence. Let $S$ be a finite subset of $V$. For any function $f$ on $\partial S$, there exists an unique function $h$ on $S \cup \partial S$ that is $p$-harmonic on $S$ and equals $f$ on $\partial S$. In the proof of existence, it was shown that the $p$-harmonic function $h$ satisfies $\min _{y \in \partial S} f(y) \leq h(x) \leq \max _{y \in \partial S} f(y)$ for all $x \in S$.

- Minimizer property. Let $h$ be a $p$-harmonic function on a finite subset $S$ of $V$. Then $I_{p}(h, S) \leq I_{p}(f, S)$ for all functions $f$ on $S \cup \partial S$ satisfying $f=h$ on $\partial S$.

- Convergence. Let $\left(S_{n}\right)$ be an increasing sequence of finite connected subsets of $V$ and let $U=\bigcup_{i} S_{i}$. Let $\left(h_{i}\right)$ be a sequence of functions on $U \cup \partial U$ such that $h_{i}(x) \rightarrow h(x)<\infty$ for every $x \in U \cup \partial U$. If $h_{i}$ is $p$-harmonic on $S_{i}$ for all $i$, then $h$ is $p$-harmonic on $U$.

- Comparison principle. Let $h$ and $u$ be $p$-harmonic functions on a finite subset $S$ of $V$. If $h \geq u$ on $\partial S$, then $h \geq u$ on $S$.

We also prove the maximum principle for bounded $p$-harmonic functions on $V$ :

Lemma 3.1. Let $h$ be a $p$-harmonic function on $V$. If there exists an $x \in V$ such that $h(x) \geq h(y)$ for all $y \in V$, then $h$ is constant on $V$.

Proof. Let $x \in V$ such that $h(x) \geq h\left(x^{\prime}\right)$ for all $x^{\prime} \in V$. Because

$$
\sum_{y \in N_{x}}|h(y)-h(x)|^{p-2} h(y)=\sum_{y \in N_{x}}|h(y)-h(x)|^{p-2} h(x),
$$

we see that $h(x)=h(y)$ for all $y \in N_{x}$. Thus $h(x)=h(z)$ for all $z \in V$ since $G$ is connected.

\section{Preliminary results}

In this section we will give some results about $\partial_{p}(G)$ and $\mathrm{BD}_{p}(G)$. Most of the results given in Propositions 4.2 through 4.8 are given in the first two sections of [Soardi 1994, Chapter VI] for the case of $p=2$. However, our presentation and some of our proofs are different. Recall that $o$ is a fixed vertex of the graph $G$. 
Lemma 4.1. If $x \in \partial_{p}(G)$ and $\left(x_{n}\right)$ is a sequence in $V$ that converges to $x$, then $d\left(o, x_{n}\right) \rightarrow \infty$ as $n \rightarrow \infty$.

Proof. Let $x \in \partial_{p}(G)$ and suppose $\left(x_{n}\right) \rightarrow x$, where $\left(x_{n}\right)$ is a sequence in $V$. Let $B$ be a positive real number. Define a function $\chi_{B}$ on $V$ by $\chi_{B}(y)=1$ if $d(o, y) \leq B$ and $\chi_{B}(y)=0$ if $d(o, y)>B$. Since $\chi_{B}$ has finite support it is an element of $\mathbb{R} G$. Suppose there exists a real number $M$ such that $d\left(o, x_{n}\right) \leq M$ for all $n$. Then $\widehat{\chi_{M}}(x)=\lim _{n \rightarrow \infty} \chi_{M}\left(x_{n}\right)=1$, a contradiction. Thus $d\left(o, x_{n}\right) \rightarrow \infty$ as $n \rightarrow \infty$.

We now characterize $p$-parabolic graphs in terms of $\partial_{p}(G)$.

Proposition 4.2. Let $G$ be a graph and let $1<p \in \mathbb{R}$. Then $\partial_{p}(G)=\varnothing$ if and only if $G$ is p-parabolic.

Proof. Assume $G$ is $p$-parabolic and suppose $\partial_{p}(G) \neq \varnothing$. Let $x \in \partial_{p}(G)$ and let $\left(x_{n}\right)$ be a sequence in $V$ that converges to $x$. Then $\widehat{1_{V}}(x)=\lim _{n \rightarrow \infty} \widehat{1_{V}}\left(x_{n}\right)=1$. By Theorem 2.2, we have $1_{V} \in B(\overline{\mathbb{R} G})_{D_{p}}$, which says that $\widehat{1_{V}}(x)=0$, a contradiction. Hence if $G$ is $p$-parabolic, then $\partial_{p}(G)=\varnothing$.

Now suppose that $G$ is $p$-hyperbolic. Then $1_{V} \notin B(\overline{\mathbb{R} G})_{D_{p}}$. Since $B(\overline{\mathbb{R} G})_{D_{p}}$ is an ideal in the commutative ring $\operatorname{BD}_{p}(G)$, there exists a maximal ideal $M$ in $\mathrm{BD}_{p}(G)$ containing $B(\overline{\mathbb{R} G})_{D_{p}}$. Using the correspondence between maximal ideals in $\mathrm{BD}_{p}(G)$ and $\operatorname{Sp}\left(\mathrm{BD}_{p}(G)\right)$, there is an $x \in \operatorname{Sp}\left(\mathrm{BD}_{p}(G)\right)$ that satisfies $\operatorname{ker}(x)=M$. So $\hat{f}(x)=x(f)=0$ for all $f \in B(\overline{\mathbb{R} G})_{D_{p}}$. For each $y \in V$, there exists an $f \in \mathbb{R} G$ (in particular $\delta_{y}$ ) such that $y(f)=f(y) \neq 0$, which means that $x$ cannot be in $V$. Also, if $x \in R_{p}(G) \backslash \partial_{p}(G)$, then there exists an $f \in B(\overline{\mathbb{R} G})_{D_{p}}$ for which $\hat{f}(x) \neq 0$. This implies that $B(\overline{\mathbb{R} G})_{D_{p}}$ is not contained in $M$. Therefore $x \in \partial_{p}(G)$.

For the rest of this paper, we will assume that $1_{V} \notin B(\overline{\mathbb{R} G})_{D_{p}}$ unless otherwise stated, that is, we assume $G$ is $p$-hyperbolic.

Let $f$ and $h$ be elements in $\operatorname{BD}_{p}(G)$ and let $1<p \in \mathbb{R}$. Define

$$
\left\langle\triangle_{p} h, f\right\rangle:=\sum_{x \in V} \sum_{y \in N_{x}}|h(y)-h(x)|^{p-2}(h(y)-h(x))(f(y)-f(x)) .
$$

This sum exists since $\sum_{x \in V} \sum_{y \in N_{x}}|| h(y)-\left.\left.h(x)\right|^{p-2}(h(y)-h(x))\right|^{q}=I_{p}(h, V)$ is finite, where $1 / p+1 / q=1$. The next few lemmas will help show the uniqueness of the decomposition of $\mathrm{BD}_{p}(G)$ that will be given in Theorem 4.6.

Lemma 4.3. Let $f_{1}$ and $f_{2}$ be functions in $D_{p}(G)$. Then $\left\langle\triangle_{p} f_{1}-\triangle_{p} f_{2}, f_{1}-f_{2}\right\rangle$ is zero if and only if $f_{1}-f_{2}$ is constant on $V$.

Proof. Let $f_{1}, f_{2} \in D_{p}(G)$ and assume there exists an $x \in V$ with a $y \in N_{x}$ such that $f_{1}(x)-f_{1}(y) \neq f_{2}(x)-f_{2}(y)$. Define a function $f:[0,1] \rightarrow \mathbb{R}$ by

$$
f(t)=\sum_{x \in V} \sum_{y \in N_{x}}\left|f_{1}(y)-f_{1}(x)+t\left(\left(f_{2}(y)-f_{2}(x)\right)-\left(f_{1}(y)-f_{1}(x)\right)\right)\right|^{p} .
$$


Observe that $f(0)=I\left(f_{1}, V\right)$ and $f(1)=I\left(f_{2}, V\right)$. A derivative calculation gives

$$
f^{\prime}(0)=p\left\langle\triangle_{p} f_{1}, f_{2}-f_{1}\right\rangle=-p\left\langle\triangle_{p} f_{1}, f_{1}-f_{2}\right\rangle .
$$

It follows from [Ekeland and Témam 1999, Proposition 5.4] that $I_{p}\left(f_{2}, V\right)>$ $I_{p}\left(f_{1}, V\right)-p\left\langle\triangle_{p} f_{1}, f_{1}-f_{2}\right\rangle$. Similarly, $I_{p}\left(f_{1}, V\right)>I_{p}\left(f_{2}, V\right)-p\left\langle\triangle_{p} f_{2}, f_{2}-f_{1}\right\rangle$. Hence, $p\left\langle\triangle_{p} f_{1}-\triangle_{p} f_{2}, f_{1}-f_{2}\right\rangle>0$ if there exists an $x \in V$ with $y \in N_{x}$ that satisfies $f_{1}(x)-f_{1}(y) \neq f_{2}(x)-f_{2}(y)$. Conversely, suppose $f_{1}-f_{2}$ is constant on $V$. We immediately see that $\left\langle\triangle_{p} f_{1}-\triangle_{p} f_{2}, f_{1}-f_{2}\right\rangle=0$.

Lemma 4.4. Let $h \in \mathrm{BD}_{p}(G)$. Then $h \in \mathrm{BHD}_{p}(G)$ if and only if $\left\langle\triangle_{p} h, \delta_{x}\right\rangle=0$ for all $x \in V$.

Proof. Let $x \in V$ and let $h \in \mathrm{BD}_{p}(G)$. The lemma follows from

$$
\left\langle\triangle_{p} h, \delta_{x}\right\rangle=-2(\operatorname{deg}(x)) \sum_{y \in N_{x}}|h(x)-h(y)|^{p-2}(h(y)-h(x)) .
$$

The lemma implies that if $h \in \mathrm{BHD}_{p}(G)$, then $\left\langle\triangle_{p} h, f\right\rangle=0$ for all $f \in \mathbb{R} G$.

Lemma 4.5. If $h \in \mathrm{BHD}_{p}(G)$ and $f \in B\left(\overline{\ell^{p}(G)}\right)_{D_{p}}$, then $\left\langle\triangle_{p} h, f\right\rangle=0$.

Proof. Let $h$ and $f$ be as stated. Then there exists a sequence $\left(f_{n}\right)$ in $\mathbb{R} G$ such that $\left\|f-f_{n}\right\|_{D_{p}} \rightarrow 0$ as $n \rightarrow \infty$ since $(\overline{\mathbb{R} G})_{D_{p}}=\left(\overline{\ell^{p}(G)}\right)_{D_{p}}$. Now

$$
\begin{aligned}
0 & \leq\left|\left\langle\triangle_{p} h, f\right\rangle\right|=\left|\left\langle\triangle_{p} h, f-f_{n}\right\rangle\right| \\
& =\left|\sum_{x \in V} \sum_{y \in N_{x}}\right| h(y)-\left.h(x)\right|^{p-2}(h(y)-h(x))\left(\left(f-f_{n}\right)(x)-\left(f-f_{n}\right)(y)\right) \mid \\
& \leq \sum_{x \in V} \sum_{y \in N_{x}}|h(y)-h(x)|^{p-1}\left|\left(f-f_{n}\right)(x)-\left(f-f_{n}\right)(y)\right| \\
& \leq\left(\sum_{x \in V} \sum_{y \in N_{x}}\left(|h(y)-h(x)|^{p-1}\right)^{q}\right)^{1 / q}\left(I_{p}\left(f-f_{n}, V\right)\right)^{1 / p} \rightarrow 0
\end{aligned}
$$

as $n \rightarrow \infty$. The last inequality follows from Hölder's inequality.

Clarkson's inequality will be needed in the next proof. Let $f_{1}$ and $f_{2}$ be elements of $D_{p}(G)$. If $2 \leq p \in \mathbb{R}$, then

$$
I_{p}\left(f_{1}+f_{2}\right)+I_{p}\left(f_{1}-f_{2}\right) \leq 2^{p-1}\left(I_{p}\left(f_{1}\right)+I_{p}\left(f_{2}\right)\right)
$$

and if $1<p \leq 2$, then

$$
\left(I_{p}\left(f_{1}+f_{2}\right)\right)^{1 /(p-1)}+\left(I_{p}\left(f_{1}-f_{2}\right)\right)^{1 /(p-1)} \leq 2\left(I_{p}\left(f_{1}\right)+I_{p}\left(f_{2}\right)\right)^{1 /(p-1)} .
$$

The following decomposition of $\operatorname{BD}_{p}(G)$ will be crucial:

Theorem 4.6. Let $1<p \in \mathbb{R}$ and suppose $f \in \mathrm{BD}_{p}(G)$. Then there exists a unique $u \in B\left(\overline{\ell^{p}(G)}\right)_{D_{p}}$ and a unique $h \in \operatorname{BHD}_{p}(G)$ such that $f=u+h$. 
Proof. Our assumption remains that $1_{V} \notin B\left(\overline{\ell^{p}(G)}\right)_{D_{p}}$. Let $f \in \mathrm{BD}_{p}(G)$. Since $f$ is bounded there exists real numbers $a$ and $b$ for which $a \leq f(x) \leq b$ is satisfied by all $x \in V$. Denote by $h_{n}$ the function that is $p$-harmonic on $B_{n}(o)$ and equal to $f$ on $V \backslash B_{n}(o)$. Because $\min _{y \in \partial B_{n}(o)} f(y) \leq h_{n}(x) \leq \max _{y \in \partial B_{n}(o)} f(y)$ for all $x \in B_{n}(o)$, we have $a \leq h_{n} \leq b$ for each $n \in \mathbb{N}$. Furthermore, if $m>n$, then $I_{p}\left(h_{m}\right) \leq I_{p}\left(h_{n}\right)$. Set $r_{n}=I_{p}\left(h_{n}\right)$ and denote the limit of the bounded decreasing sequence $\left(r_{n}\right)$ by $r$. We are still assuming that $m>n$. By the minimizing property of $p$-harmonic functions, $I_{p}\left(h_{m}, V\right) \leq I_{p}\left(\left(h_{n}+h_{m}\right) / 2, V\right)$ since $\left(h_{n}+h_{m}\right) / 2=h_{m}$ on $V \backslash B_{m}(o)$. Using Clarkson's inequality we obtain for $2 \leq p \in \mathbb{R}$,

$$
\begin{aligned}
r_{m} & \leq I_{p}\left(\frac{1}{2}\left(h_{n}+h_{m}\right), V\right) \\
& \leq I_{p}\left(\frac{1}{2}\left(h_{n}+h_{m}\right), V\right)+I_{p}\left(\frac{1}{2}\left(h_{n}-h_{m}\right), V\right) \\
& \leq 2^{p-1}\left(I_{p}\left(\frac{1}{2} h_{n}, V\right)+I_{p}\left(\frac{1}{2} h_{m}, V\right)\right) \\
& =\frac{1}{2}\left(I_{p}\left(h_{n}, V\right)+I_{p}\left(h_{m}, V\right)\right)
\end{aligned}
$$

and for $1<p \leq 2$,

$$
\begin{aligned}
r_{m}^{1 /(p-1)} & \leq\left(I_{p}\left(\frac{1}{2}\left(h_{n}+h_{m}\right), V\right)\right)^{1 /(p-1)} \\
& \leq\left(I_{p}\left(\frac{1}{2}\left(h_{n}+h_{m}\right), V\right)\right)^{1 /(p-1)}+\left(I_{p}\left(\frac{1}{2}\left(h_{n}-h_{m}\right), V\right)\right)^{1 /(p-1)} \\
& \leq 2\left(I_{p}\left(\frac{1}{2} h_{n}, V\right)+I_{p}\left(\frac{1}{2} h_{m}, V\right)\right)^{1 /(p-1)} .
\end{aligned}
$$

Letting $m, n \rightarrow \infty$, we have $I_{p}\left(\frac{1}{2}\left(h_{n}+h_{m}\right), V\right) \rightarrow r$ and $I_{p}\left(\frac{1}{2}\left(h_{n}-h_{m}\right), V\right) \rightarrow 0$. Also, $\left(\left|h_{n}(o)\right|\right)$ is a bounded sequence; thus $\left(h_{n}\right)$ is a Cauchy sequence in $D_{p}(G)$. Set $h$ equal to the limit function of the sequence $\left(h_{n}\right)$ in $D_{p}(G)$. Because $\left(h_{n}\right)$ also converges pointwise to $h$, the convergence property says that $h$ is $p$-harmonic. Clearly, $a \leq h \leq b$ on $V$, so $h \in \operatorname{BHD}_{p}(G)$. Let $u$ be the limit function in $D_{p}(G)$ of the Cauchy sequence $\left(f-h_{n}\right)$. Since $f-h_{n} \in \mathbb{R} G$ for each $n$, we see that $u \in B(\overline{\mathbb{R} G})_{D_{p}}$. Thus $f=u+h$.

To show that this decomposition is unique, suppose $f=u_{1}+h_{1}=u_{2}+h_{2}$, where $u_{1}, u_{2} \in B\left(\overline{\ell^{p}(G)}\right)_{D_{p}}$ and $h_{1}, h_{2} \in \mathrm{BHD}_{p}(G)$. Lemma 4.5 says that

$$
\left\langle\triangle_{p} h_{1}-\triangle_{p} h_{2}, h_{1}-h_{2}\right\rangle=\left\langle\triangle_{p} h_{1}-\triangle_{p} h_{2}, u_{2}-u_{1}\right\rangle=0
$$

since $u_{1}-u_{2} \in B\left(\overline{\ell^{p}(G)}\right)_{D_{p}}$. However, $u_{1}-u_{2}=0$ since $1_{V} \notin B\left(\overline{\ell^{p}(G)}\right)_{D_{p}}$.

Theorem 4.7 (maximum principle). Let $h$ be a nonconstant function in $\operatorname{BHD}_{p}(G)$ and suppose $a$ and $b$ are real numbers for which $a \leq \hat{h} \leq b$ on $\partial_{p}(G)$. Then $a<h<b$ on $V$.

Proof. Since $\hat{h}$ is continuous on the compact space $\operatorname{Sp}\left(\mathrm{BD}_{p}(G)\right)$, there is a number $c>0$ such that $b-\hat{h} \geq-c$ on $\operatorname{Sp}\left(\operatorname{BD}_{p}(G)\right)$. Let $\epsilon>0$ and set $F_{\epsilon}$ to be the set of $x \in \operatorname{Sp}\left(\operatorname{BD}_{p}(G)\right)$ such that $b-h+\epsilon \leq 0$. To prove the theorem, we will first show 
that there exists an $f \in B(\overline{\mathbb{R} G})_{D_{p}}$ with $\hat{f}=1$ on $F_{\epsilon}$ and $0 \leq \hat{f} \leq 1$ on $\operatorname{Sp}\left(\operatorname{BD}_{p}(G)\right)$. This $f$ will yield the inequality

$$
c f+b-h+\epsilon \geq 0 \quad \text { on } \operatorname{Sp}\left(\operatorname{BD}_{p}(G)\right) .
$$

We will then show that $b-h+\epsilon \geq 0$ on $V$. Combining this with Lemma 3.1 and the assumption that $h$ is nonconstant will give $h<b$ on $V$.

Observe that $F_{\epsilon} \cap \partial_{p}(G)=\varnothing$ and $F_{\epsilon}$ is a closed subset of $\operatorname{Sp}\left(\mathrm{BD}_{p}(G)\right)$. For each $x \in F_{\epsilon}$ there exists an $f_{x} \in B(\overline{\mathbb{R} G})_{D_{p}}$ for which $\hat{f}_{x}(x) \neq 0$. Since $B(\overline{\mathbb{R} G})_{D_{p}}$ is an ideal, we may assume that $f_{x} \geq 0$ on $V$ and $\hat{f}_{x}(x)>0$. Let $U_{x}$ be a neighborhood of $x$ in $\operatorname{Sp}\left(\operatorname{BD}_{p}(G)\right)$ that satisfies $f_{x}(y)>0$ for all $y \in U_{x}$. By compactness there exists $x_{1}, \ldots, x_{n}$ for which $F_{\epsilon} \subseteq \bigcup_{j=1}^{n} U_{x_{j}}$. Set

$$
g=\sum_{j=1}^{n} f_{x_{j}} \quad \text { and } \quad \alpha=\inf \left\{g(x) \mid x \in F_{\epsilon}\right\} .
$$

Clearly $\alpha>0$ and $g \in B(\overline{\mathbb{R} G})_{D_{p}}$. Now define a function $f$ on $\operatorname{Sp}\left(\operatorname{BD}_{p}(G)\right)$ by $f=\min \left(1, \alpha^{-1} g\right)$. Note that $0 \leq \hat{f} \leq 1$ on $\operatorname{Sp}\left(\operatorname{BD}_{p}(G)\right)$ and $\hat{f}=1$ and $F_{\epsilon}$. We still need to show that $f \in B(\overline{\mathbb{R} G})_{D_{p}}$. Let $\left(g_{n}\right)$ be a sequence in $\mathbb{R} G$ that converges to $g$ in $D_{p}(G)$, so $I_{p}\left(\left(g-g_{n}\right), V\right) \rightarrow 0$ as $n \rightarrow \infty$. Set $f_{n}=\min \left(1, \alpha^{-1} g_{n}\right)$. The sequence $\left(f_{n}\right)$ converges pointwise to $f$. Furthermore, by passing to a subsequence if necessary, $\left(f_{n}\right)$ converges weakly to a function $\bar{f}$ in $D_{p}(G)$ since $I_{p}\left(f_{n}, V\right)$ is bounded. Clearly $\bar{f}$ is bounded, so $\bar{f} \in B(\overline{\mathbb{R} G})_{D_{p}}$. It is also true $\left(f_{n}\right)$ converges pointwise to $\bar{f}$ because point evaluations by elements of $V$ are continuous linear functionals on $\mathrm{BD}_{p}(G)$. Hence, $\bar{f}=f$ and $f \in \mathrm{BD}_{p}(G)$. Inequality (4-1) is now established.

Next we will show that $b-h+\epsilon \geq 0$ on $V$. Put $v_{\epsilon}=c f+b-h+\epsilon$ and denote by $h_{n}$ the unique function that is $p$-harmonic on $B_{n}(o)$ and agrees with $v_{\epsilon}$ on $V \backslash B_{n}(o)$. We claim that $h_{n} \geq 0$ on $B_{n}(o)$. Supposing otherwise, there exists an $x \in B_{n}(o)$ for which $h_{n}(x)<0$. Define a function $h_{n}^{*}$ by

$$
h_{n}^{*}= \begin{cases}v_{\epsilon} & \text { if } x \in V \backslash B_{n}(o), \\ \max \left(h_{n}, 0\right) & \text { if } x \in B_{n}(o) .\end{cases}
$$

Now $I_{p}\left(h_{n}^{*}, B_{n}(o)\right)<I_{p}\left(h_{n}, B_{n}(o)\right)$, but this contradicts the minimizer property of $p$-harmonic functions. This proves the claim. By using the argument used in the proof of Theorem 4.6, we see that $\left(h_{n}\right)$ converges to a bounded $p$-harmonic function $\bar{h}$ and that there exists a $v \in B(\overline{\mathbb{R} G})_{D_{p}}$ such that $v_{\epsilon}=v+\bar{h}$. Furthermore $\bar{h} \geq 0$ on $V$ because $h_{n} \geq 0$ for each $n$. The uniqueness part of Theorem 4.6 says that $v=c f$ and $\bar{h}=b-h+\epsilon$. Hence $b \geq h-\epsilon$ on $V$. Thus $h<b$ on $V$.

A similar argument shows that $a<h$ on $V$. Therefore, $a<h<b$ on $V$.

We now characterize the functions in $\operatorname{BD}_{p}(G)$ that vanish on $\partial_{p}(G)$. 
Theorem 4.8. Let $f \in \mathrm{BD}_{p}(G)$. Then $f \in B\left(\overline{\left(\ell^{p}(G)\right.}\right)_{D_{p}}$ if and only if $\hat{f}(x)=0$ for all $x \in \partial_{p}(G)$.

Proof. Since $B\left(\overline{\ell^{p}(G)}\right)_{D_{p}}=B(\overline{\mathbb{R} G})_{D_{p}}$ it follows immediately that $\hat{f}(x)=0$ for all $f \in B\left(\overline{\ell^{p}(G)}\right)_{D_{p}}$ and all $x \in \partial_{p}(G)$.

Conversely, suppose $f \in \mathrm{BD}_{p}(G)$ and $\hat{f}(x)=0$ for all $x \in \partial_{p}(G)$. Theorem 4.6 allows us to write $f=u+h$, where $u \in B\left(\overline{\ell^{p}(G)}\right)_{D_{p}}$ and $h \in \operatorname{BHD}_{p}(G)$. Now $\hat{h}(x)=0$ for all $x \in \partial_{p}(G)$ since $\hat{u}(x)=0$. Therefore, $h=0$ by the maximum principle.

Corollary 4.9. Every function in $\operatorname{BHD}_{p}(G)$ is uniquely determined by its values on $\partial_{p}(G)$.

Proof. Let $h_{1}$ and $h_{2}$ be elements of $\operatorname{BHD}_{p}(G)$ with $\widehat{h_{1}}(x)=\widehat{h_{2}}(x)$ for all $x \in \partial_{p}(G)$. Then $h_{1}-h_{2} \in B\left(\overline{\ell^{p}(G)}\right)_{D_{p}}$. Let $\left(f_{n}\right)$ be a sequence in $\ell^{p}(G)$ that converges to $h_{1}-h_{2}$. Using Lemma 4.5 , we obtain

$$
\left\langle\triangle_{p} h_{1}-\triangle_{p} h_{2}, h_{1}-h_{2}\right\rangle=\lim _{n \rightarrow \infty}\left\langle\triangle_{p} h_{1}-\triangle_{p} h_{2}, f_{n}\right\rangle=0 .
$$

It now follows from Lemma 4.3 that $h_{1}-h_{2}=0$.

We can now characterize when $\operatorname{BHD}_{p}(G)$ is precisely the constant functions.

Theorem 4.10. Let $1<p \in \mathbb{R}$. Then $\operatorname{BHD}_{p}(G) \neq \mathbb{R}$ if and only if $\#\left(\partial_{p}(G)\right)>1$.

Proof. Suppose that $\#\left(\partial_{p}(G)\right)=1$ and that $x \in \partial_{p}(G)$. Let $h \in \operatorname{BHD}_{p}(G)$. Then $\hat{h}(x)=c$ for some constant $c$. It follows from Corollary 4.9 that the function $h(x)=c$ for all $x \in V$ is the only function in $\operatorname{BHD}_{p}(G)$ with $\hat{h}(x)=c$. Hence $\mathrm{BHD}_{p}(G)=\mathbb{R}$.

Conversely, suppose \# $\left(\partial_{p}(G)\right)>1$. Let $x, y \in \partial_{p}(G)$ such that $x \neq y$ and pick an $f \in \mathrm{BD}_{p}(G)$ that satisfies $x(f) \neq y(f)$. By Theorem 4.8, $f \notin B\left(\overline{\ell^{p}(G)}\right)_{D_{p}}$. It now follows from Theorem 4.6 and Theorem 4.8 that there exists an $h \in \operatorname{BHD}_{p}(G)$ with $\hat{h}(z)=\hat{f}(z)$ for all $z \in \partial_{p}(G)$. Since $V$ is dense in $\operatorname{Sp}\left(\operatorname{BD}_{p}(G)\right)$, there exist sequences $\left(x_{n}\right)$ and $\left(y_{n}\right)$ in $V$ such that $\left(x_{n}\right)(h) \rightarrow x(h)$ and $\left(y_{n}\right)(h) \rightarrow y(h)$. Hence $\lim _{n \rightarrow \infty} h\left(x_{n}\right)=x(h) \neq y(h)=\lim _{n \rightarrow \infty} h\left(y_{n}\right)$. Hence $h$ is not constant on $V$.

We now define the important concept of a $D_{p}$-massive subset of a graph. An infinite connected subset $U$ of $V$ with $\partial U \neq \varnothing$ is called a $D_{p}$-massive subset if there exists a nonnegative function $u \in \mathrm{BD}_{p}(G)$ such that

(a) $\Delta_{p} u(x)=0$ for all $x \in U$,

(b) $u(x)=0$ for $x \in \partial U$, and

(c) $\sup _{x \in U} u(x)=1$.

We call any $u$ that satisfies these conditions an inner potential of the $D_{p}$-massive subset $U$. The following will be needed in the proof of Lemma 5.1. 
Proposition 4.11. If $U$ is a $D_{p}$-massive subset of $V$, then $\overline{i(U)}$ contains at least one point of $\partial_{p}(G)$.

Proof. We will write $\bar{U}$ for $\overline{i(U)}$, where the closure is taken in $\left.\operatorname{Sp} \operatorname{BD}_{p}(G)\right)$. Assume $\bar{U} \cap \partial_{p}(G)=\varnothing$ and let $u$ be an inner potential for $U$. We may and do assume that $u=0$ on $V \backslash U$. By the existence property for $p$-harmonic functions, there exists a $p$-harmonic function $h_{n}$ on $B_{n}(o)$ such that $h_{n}=u$ on $\partial B_{n}(o)$ for each natural number $n$. Also $0 \leq \min _{y \in \partial B_{n}(o)} u(y) \leq h_{n} \leq \max _{y \in \partial B_{n}(o)} u(y) \leq 1$ on $B_{n}(o)$. Extend $h_{n}$ to all of $V$ by setting $h_{n}=u$ on $V \backslash B_{n}(o)$. By the minimizing property of $p$-harmonic functions, $I_{p}\left(h_{n}, B_{n}(o)\right) \leq I_{p}\left(u, B_{n}(o)\right)$, and so $I_{p}\left(h_{n}, V\right) \leq I_{p}(u, V)$. Both $h_{n}$ and $u$ are $p$-harmonic on $U \cap B_{n}(o)$, and we have $u(x) \leq h_{n}(x)$ for all $x \in \partial\left(U \cap B_{n}(o)\right)$. The comparison principle says that $u \leq h_{n}$ on $U \cap B_{n}(o)$. On $B_{n}(o) \backslash U$ we have $u=0$, so $u \leq h_{n} \leq 1$ for each $n$. By taking a subsequence if needed, we assume that $\left(h_{n}\right)$ converges pointwise to a function $h$. Now $u \leq h \leq 1$ on $V$, so $\sup _{x \in U} h(x)=1$. By the convergence property for $p$-harmonic functions, $h$ is $p$-harmonic and $h \in \operatorname{BHD}_{p}(G)$ since $I_{p}\left(h_{n}, V\right) \leq I_{p}(u, V)<\infty$ for all $n$.

Let $x \in \partial_{p}(G)$. Since $u-h_{n}=0$ on $V \backslash B_{n}(o)$, we see that $\hat{u}(x)-\widehat{h_{n}}(x)=0$ for all $n$; thus $\overline{u-h}=0$ on $\partial_{p}(G)$. According to Theorem 4.8, $u-h \in B\left(\overline{\ell^{p}(G)}\right)_{D_{p}}$. Hence $u=f+h$, where $f \in B\left(\overline{\ell^{p}(G)}\right)_{D_{p}}$. Another appeal to Theorem 4.8 shows that $\hat{u}=\hat{h}$ on $\partial_{p}(G)$. If $x \in \partial_{p}(G)$, then $\hat{u}(x)=0$ because if $\left(x_{n}\right)$ is a sequence in $V$ converging to $x$, then $u\left(x_{n}\right)=0$ for all but a finite number of $n$ since we are assuming $\bar{U} \cap \partial_{p}(G)=\varnothing$. So $\hat{h}(x)=0$ for all $x \in \partial_{p}(G)$. Hence $h=0$ on $V$ by the maximum principle, which contradicts $\sup _{U} h=1$. Therefore, if $U$ is a $D_{p}$-massive subset of $V$, then $\bar{U}$ contains at least one point of $\partial_{p}(G)$.

It would be nice to know if the converse of Proposition 4.11 is true. That is, if $x \in \partial_{p}(G)$, does there exist a $D_{p}$-massive subset $U$ of $V$ such that $x \in \bar{U}$ ? The next result leads to a partial converse and also describes a base of neighborhoods for open sets in $\partial_{p}(G)$.

Proposition 4.12. Let $x \in \partial_{p}(G)$ and let $O$ be an open set in $\partial_{p}(G)$ containing $x$. Then there exists a subset $U$ of $V$ such that

(a) $U=\bigcup_{\alpha \in I} A_{\alpha}$, where each $A_{\alpha}$ is a $D_{p}$-massive subset of $V$ and $I$ is an index set, and $A_{\alpha} \cap A_{\beta}=\varnothing$ if $\alpha \neq \beta$, and

(b) $x \in \bar{U} \cap \partial_{p}(G) \subseteq O$.

Proof. Let $x \in \partial_{p}(G)$, and let $O$ be an open set of $\partial_{p}(G)$ containing $x$. By Urysohn's lemma there exists an $\left.f \in C\left(\operatorname{Sp}_{\left(B_{p}\right.}(G)\right)\right)$ with $0 \leq f \leq 1, f(x)=1$ and $f=0$ on $\partial_{p}(G) \backslash O$. Since the Gelfand transform of $\operatorname{BD}_{p}(G)$ is dense in $C\left(\operatorname{Sp}\left(\operatorname{BD}_{p}(G)\right)\right)$ with respect to the supremum norm, we will assume $f \in \mathrm{BD}_{p}(G)$. By Theorem 4.6 we have the decomposition $f=w+h$, where $w \in B\left(\overline{\ell^{p}(G)}\right)_{D_{p}}$ and $h \in \operatorname{BHD}_{p}(G)$. Since $\hat{w}=0$ on $\partial_{p}(G)$, it follows that $\hat{h}(x)=1$ and $\hat{h}=0$ 
on $\partial_{p}(G) \backslash O$. Also, $0 \leq \hat{h} \leq 1$ on $\partial_{p}(G)$, so $0<h<1$ on $V$ by the maximum principle and $0 \leq \hat{h} \leq 1$ on $\operatorname{Sp}\left(\operatorname{BD}_{p}(G)\right)$ due to the density of $V$. Fix $\epsilon$ with $0<\epsilon<1$ and set $U=\{x \in V \mid h(x)>\epsilon\}$. Let $A$ be a component of $U$. It now follows from the comparison principle that $A$ is infinite. Define a function $v$ on $V$ by $v=(h-\epsilon) /(1-\epsilon)$. There exists a $p$-harmonic function $u_{n}$ on $B_{n}(o) \cap A$ taking the values $\max \{0, v\}$ on $V \backslash\left(B_{n}(o) \cap A\right)$ and such that $0 \leq u_{n} \leq 1$ on $B_{n}(o) \cap A$. By passing to a subsequence if necessary, we may assume that $\left(u_{n}\right)$ converges pointwise to a function $u$. By the convergence property, $u$ is $p$-harmonic on $A$. Also $v \leq u_{n} \leq 1$ on $B_{n}(o)$, so by replacing $u$ by a suitable scalar multiple if necessary, we have $\sup _{a \in A} u(a)=1$. Also, $u=0$ on $\partial A$ because $h \leq \epsilon$ on $\partial A$. Since $h \in \mathrm{BD}_{p}(G)$, it follows that $u \in \mathrm{BD}_{p}(G)$. Thus $A$ is a $D_{p}$-massive subset with inner potential $u$. Hence, each component of $U$ is a $D_{p}$-massive subset in $V$. So $U=\bigcup_{\alpha \in I} A_{\alpha}$, where each $A_{\alpha}$ is $D_{p}$-massive. The proof of part (a) is complete.

Clearly $x \in \bar{U}$. We will show that $\bar{U} \cap \partial_{p}(G) \subseteq O$. Let $y \in \bar{U} \cap \partial_{p}(G)$ and let $\left(y_{k}\right)$ be a sequence in $U$ that converges to $y$. Then $f(y)=\hat{h}(y)=\lim _{k \rightarrow \infty} h\left(y_{k}\right) \geq \epsilon$. Hence $y \in O$ since $f=0$ on $\partial_{p}(G) \backslash O$.

The following partial converse to Proposition 4.11 is a direct consequence of Proposition 4.12.

Corollary 4.13. If \# $\left(\partial_{p}(G)\right)$ is finite, then for each $x \in \partial_{p}(G)$ there exists a $D_{p}$-massive subset $U$ of $V$ such that $x \in \bar{U}$.

\section{Proofs of Theorem 2.4 and Theorem 2.6}

The key ingredient in the proof of Theorem 2.4 is the following.

Lemma 5.1. Let $1<p \in \mathbb{R}$ and suppose that $G$ is a p-parabolic graph. If $f$ is a nonconstant function in $\operatorname{BHD}_{p}(G)$, then $\sup _{V} f>\lim \sup _{d(o, x) \rightarrow \infty} f$.

Proof. Suppose that $\lim \sup _{d(o, x) \rightarrow \infty} f(x)=\sup _{V} f=M$. Since $f$ is nonconstant, there exists an $\epsilon>0$ such that the set $W=\{x \in V \mid f(x)>M-\epsilon\}$ is a proper infinite subset of $V$. Let $U$ be a component of $W$. If $U$ is finite, then we can construct a unique $p$-harmonic function $w$ on $U$ that agrees with $f$ on $\partial U$. Since $f$ is $p$-harmonic, $f=w$ on $U$ by uniqueness. But if $x \in U$, then

$$
w(x) \leq \max _{y \in \partial U} f(y) \leq M-\epsilon<f(x),
$$

a contradiction. Thus $U$ is infinite. Now set $h=(f-M+\epsilon) / \epsilon$. There is an number $N \in \mathbb{N}$ such that $B_{n}(o) \cap U \neq \varnothing$ for $n>N$. For $n>N$, let $u_{n}$ be a $p$-harmonic function on $B_{n}(o) \cap U$ that takes the values $\max \{0, h\}$ on $V \backslash\left(B_{n}(o) \cap U\right)$. Note that $u_{n} \geq 0$. Since $h$ is $p$-harmonic on $B_{n}(o) \cap U$, it follows from the comparison principle that $h \leq u_{n} \leq 1$ on $B_{n}(o) \cap U$. By taking a subsequence if necessary, we may assume that the sequence $\left(u_{n}\right)$ converges pointwise to a function $u$. By 
the convergence property, $u$ is $p$-harmonic on $U$. If $x \in \partial U$, then $f(x) \leq M-\epsilon$. Therefore, $u_{n}(x)=0$ for all $n$, which implies $u(x)=0$. Thus $u=0$ on $\partial U$. Since $\sup _{U} h=1$, we see that $\sup _{U} u=1$. We can show using the minimizing property for $p$-harmonic functions that $I_{p}\left(u_{n}, U \cap B_{n}(o)\right) \leq I_{p}\left(\max \{0, h\}, U \cap B_{n}(o)\right)$, and it follows from this inequality that $I_{p}\left(u_{n}, U\right) \leq I_{p}(h, U)$. Hence $I_{p}(u, U)<\infty$ because $I_{p}(h, V)<\infty$. Thus $U$ is a $D_{p}$-massive subset of $V$.

By Proposition 4.11, we have $\bar{U} \cap \partial_{p}(G) \neq \varnothing$, which contradicts Proposition 4.2 since we are assuming $G$ is $p$-parabolic. Hence $\sup _{V} f>\limsup _{d(o, x) \rightarrow \infty} f$.

Proof of Theorem 2.4. Let $h \in \mathrm{BHD}_{p}(G)$ and suppose that $h$ is nonconstant. Since $h$ is bounded, $\sup _{V} h=B<\infty$. Lemma 5.1 says that there exists an $x \in V$ such that $h(x)=B$. By the maximum principle, $h$ is constant on $V$, a contradiction. Hence $\operatorname{BHD}_{p}(G)$ consists of only the constant functions. Therefore, $\operatorname{HD}_{p}(G)$ is precisely the constant functions by [Holopainen and Soardi 1997a, Lemma 4.4].

Proof of Theorem 2.6. Let $f$ be a continuous function on $\partial_{p}(G)$. By Tietze's extension theorem, there exists a continuous extension of $f$, which we also denote by $f$, to all of $\operatorname{Sp}\left(\operatorname{BD}_{p}(G)\right)$. Let $\left(f_{n}\right)$ be a sequence in $\operatorname{BD}_{p}(G)$ converging to $f$ in the supremum norm. For each $n \in \mathbb{N}$ and each $r \in \mathbb{N}$, let $h_{n, r}$ be a function on $V$ that is $p$-harmonic on $B_{r}(o)$ and takes the values $f_{n}$ on $V \backslash B_{r}(o)$. The function $h_{n, r} \in \mathrm{BD}_{p}(G)$ since $B_{r}(o)$ is finite, and $\left|h_{n, r}\right| \leq \sup _{V}\left|f_{n}\right|$ because

$$
\min _{y \in \partial B_{r}(o)} f_{n}(y) \leq h_{n, r} \leq \max _{y \in \partial B_{r}(o)} f_{n}(y) \quad \text { on } B_{r}(o) .
$$

By the Ascoli-Arzela theorem, there exists a subsequence of $\left(h_{n, r}\right)$, which we also denote by $\left(h_{n, r}\right)$, that converges uniformly on all finite subsets of $V$ to a function $h_{n}$ as $r$ goes to infinity. The function $h_{n}$ is $p$-harmonic on $V$ by the convergence property. For each $r$, the minimizing property of $p$-harmonic functions gives $I_{p}\left(h_{n, r}, B_{r}(o)\right) \leq I_{p}\left(f_{n}, B_{r}(o)\right)$, so $I_{p}\left(h_{n, r}, V\right) \leq I_{p}\left(f_{n}, V\right)$, which implies $h_{n} \in \operatorname{BHD}_{p}(G)$.

Let $\epsilon>0$. Since $\left(f_{n}\right) \rightarrow f$ in the supremum norm, there exists a number $N$ such that $\sup _{V}\left|f_{n}-f_{m}\right|<\epsilon$ for $n, m \geq N$. It follows that $\sup _{\partial B_{r}(o)}\left|h_{n, r}-h_{m, r}\right|<$ $\epsilon$ for all $r \in \mathbb{N}$ because $f_{n}=h_{n, r}$ on $V \backslash B_{r}(o)$. Both $h_{n, r}$ and $h_{m, r}+\epsilon$ are $p$-harmonic on $B_{r}(o)$ and $h_{m, r}-\epsilon \leq h_{n, r} \leq h_{m, r}+\epsilon$ on $\partial B_{r}(o)$, so by applying the comparison principle, we obtain $\sup _{B_{r}(o)}\left|h_{n, r}-h_{m, r}\right|<\epsilon$ for all $r$. It now follows that $\sup _{B_{r}(o)}\left|h_{n}-h_{m}\right|<3 \epsilon$ for all $r$. Thus $\sup _{V}\left|h_{n}-h_{m}\right| \leq 3 \epsilon$. Hence, the Cauchy sequence $\left(h_{n}\right)$ converges uniformly on finite subsets of $V$ to a function $h$, which is $p$-harmonic by the convergence property.

Let $\epsilon>0$. There exists an $N \in \mathbb{N}$ such that $\sup _{V}\left|f_{n}-f\right|<\epsilon$ and $\sup _{V}\left|h_{n}-h\right|<\epsilon$ if $n \geq N$. Let $x \in \partial_{p}(G)$. Since $f_{n}(x)=h_{n}(x)$, there exists a neighborhood $U$ of $x$ such that $\left|h_{n}(y)-f_{n}(x)\right|<\epsilon$ for all $y \in U$. Therefore, $\lim _{k \rightarrow \infty} h\left(x_{k}\right)=f(x)$, where $\left(x_{k}\right)$ is a sequence in $V$ that converges to $x$. 


\section{Proofs of Theorem 2.8 and Theorem 2.9}

Let $G$ and $H$ be graphs with vertex sets $V_{G}$ and $V_{H}$, respectively. Fix a vertex $o_{G}$ in $G$ and a vertex $o_{H}$ in $H$. Let $\phi: G \rightarrow H$ be a rough isometry, and let $\phi^{*}$ denote the map from $\ell^{\infty}(H)$ to $\ell^{\infty}(G)$ given by $\phi^{*} f(x)=f(\phi(x))$. We start by defining a map $\bar{\phi}: \partial_{p}(G) \rightarrow \partial_{p}(H)$. Let $x \in \partial_{p}(G)$. Then there exists a sequence $\left(x_{n}\right)$ in $V_{G}$ such that $\left(x_{n}\right) \rightarrow x$. Now $\left(\phi\left(x_{n}\right)\right)$ is a sequence in the compact Hausdorff space $\operatorname{Sp}\left(\mathrm{BD}_{p}(H)\right)$. By passing to a subsequence, if necessary we may assume that $\left(\phi\left(x_{n}\right)\right)$ converges to a unique limit $y$ in $\operatorname{Sp}\left(\operatorname{BD}_{p}(H)\right)$. Now define $\bar{\phi}(x)=y$. Before we show that $y \in \partial_{p}(H)$ and $\bar{\phi}$ is well defined, we need a lemma.

Lemma 6.1. Let $G$ and $H$ be graphs. If $\phi: G \rightarrow H$ is a rough isometry, then

(a) $\phi^{*}$ maps $\mathrm{BD}_{p}(H)$ to $\mathrm{BD}_{p}(G)$,

(b) $\phi^{*}$ maps $\ell^{p}(H)$ to $\ell^{p}(G)$, and

(c) $\phi^{*}$ maps $B\left(\overline{\ell^{p}(H)}\right)_{D_{p}}$ to $B\left(\overline{\ell^{p}(G)}\right)_{D_{p}}$.

Proof. We will only prove part (a) since the proofs of parts (b) and (c) are similar. Let $f \in \mathrm{BD}_{p}(H)$. We will now show that $\phi^{*} f \in \mathrm{BD}_{p}(G)$. Let $x \in V_{G}$ and $w \in N_{x}$, so $x$ and $w$ are neighbors in $G$ but $\phi(w)$ and $\phi(x)$ are not necessarily neighbors in $H$. However, by the definition of rough isometry there exists constants $a \geq 1$ and $b \geq 0$ such that $d_{H}(\phi(w), \phi(x)) \leq a+b$. Set $h_{1}=\phi(x)$ and $h_{l}=\phi(w)$, and let $h_{1}, \ldots, h_{l}$ be a path in $H$ with length at most $a+b$. Thus

$$
\left|\phi^{*} f(w)-\phi^{*} f(x)\right|^{p}=|f(\phi(w))-f(\phi(x))|^{p}
$$

$$
\leq|a+b|^{p-1} \sum_{j=1}^{l-1}\left|f\left(h_{j+1}\right)-f\left(h_{j}\right)\right|^{p} .
$$

The inequality follows from Jensen's inequality applied to the function $x^{p}$ for $x>0$.

Let $y \in V_{H}$ and $z \in N_{y}$. We claim that there is at most a finite number of paths in $H$ of length at most $a+b$ that contain the edge $y, z$ and have the endpoints $\phi(x)$ and $\phi(w)$. To see this, let $U$ be the set of all elements in $V_{G}$ such that the four distances $d_{H}(\phi(x), y), d_{H}(\phi(x), z), d_{H}(\phi(w), y)$ and $d_{H}(\phi(w), z)$ are all at most $a+b$. Let $x, x^{\prime} \in U$. By the triangle inequality, $d_{H}\left(\phi\left(x^{\prime}\right), \phi(x)\right) \leq$ $d_{H}\left(\phi\left(x^{\prime}\right), y\right)+d_{H}(\phi(x), y)$. It now follows from the definition of rough isometry that $d_{G}\left(x^{\prime}, x\right) \leq 2 a^{2}+3 a b$. Thus the metric ball $B\left(x, 2 a^{2}+3 a b+1\right)$ contains $U$ as a subset. Hence the cardinality of $U$ is bounded above by some constant $k$, which is independent of $y$ and $z$. Since $f \in \mathrm{BD}_{p}(H)$ it follows from (6-1) that

$$
\sum_{x \in V_{G}} \sum_{w \in N_{x}}\left|\phi^{*} f(w)-\phi^{*} f(x)\right|^{p} \leq|a+b|^{p-1} k \sum_{y \in V_{H}} \sum_{z \in N_{y}}|f(z)-f(y)|^{p}<\infty .
$$

Proposition 6.2. The map $\bar{\phi}$ is well defined from $\partial_{p}(G)$ to $\partial_{p}(H)$. 
Proof. Let $x, y$ and $\left(x_{n}\right)$ be as above. We first show that $y \in \partial_{p}(H)$. Lemma 4.1 tells us that $d_{G}\left(o_{G}, x_{n}\right) \rightarrow \infty$ as $n \rightarrow \infty$. The element $\phi\left(o_{G}\right)$ is fixed in $H$, so it follows from the definition of rough isometry that $d_{H}\left(\phi\left(o_{G}\right), \phi\left(x_{n}\right)\right) \rightarrow \infty$ as $n \rightarrow \infty$. Thus $y \in \operatorname{Sp}\left(\operatorname{BD}_{p}(H)\right) \backslash H$ since $y=\lim _{n \rightarrow \infty} \phi\left(x_{n}\right) \notin H$. Let $f \in B\left(\overline{\ell^{p}(H)}\right)_{D_{p}}$ and suppose $\hat{f}(y) \neq 0$. Then $0 \neq \lim _{n \rightarrow \infty} f\left(\phi\left(x_{n}\right)\right)=\phi^{*} f(x)$. By Lemma 6.1(c), $\phi^{*} f \in B\left(\overline{\ell^{p}(G)}\right)_{D_{p}}$ and Theorem 4.8 says that $\phi^{*} f(x)=0$, a contradiction. Hence $\hat{f}(y)=0$ for all $f \in B\left(\overline{\ell^{p}(H)}\right)_{D_{p}}$, so $y \in \partial_{p}(H)$.

We will now show that $\bar{\phi}$ is well-defined. Let $\left(x_{n}\right)$ and $\left(x_{n}^{\prime}\right)$ be sequences in $V_{G}$ that both converge to $x \in \partial_{p}(G)$. Now suppose that $\left(\phi\left(x_{n}\right)\right)$ converges to $y_{1}$ and $\left(\phi\left(x_{n}^{\prime}\right)\right)$ converges to $y_{2}$ in $\operatorname{Sp}\left(\mathrm{BD}_{p}(H)\right)$. Assume that $y_{1} \neq y_{2}$ and let $f \in \mathrm{BD}_{p}(H)$ such that $f\left(y_{1}\right) \neq f\left(y_{2}\right)$. By Lemma 6.1(a), we have $\phi^{*} f \in \mathrm{BD}_{p}(G)$. Thus

$$
\lim _{n \rightarrow \infty} \phi^{*} f\left(x_{n}\right)=\phi^{*} f(x)=\lim _{n \rightarrow \infty} \phi^{*} f\left(x_{n}^{\prime}\right),
$$

which implies $f\left(y_{1}\right)=f\left(y_{2}\right)$, a contradiction. Hence $\bar{\phi}$ is a well-defined map from $\partial_{p}(G)$ to $\partial_{p}(H)$.

The next lemma will be used to show that $\bar{\phi}$ is one-to-one and onto.

Lemma 6.3. Let $\phi: G \rightarrow H$ be a rough isometry and let $\psi$ be a rough inverse of $\phi$. If $f \in D_{p}(G)$, then $\lim _{d_{G}\left(o_{G}, x\right) \rightarrow \infty}|f((\psi \circ \phi)(x))-f(x)|=0$.

Proof. Let $x \in V_{G}$. Since $\psi$ is a rough inverse of $\phi$, there are nonnegative constants $a, b$ and $c$ with $a \geq 1$ such that $d_{G}((\psi \circ \phi)(x), x) \leq a(c+b)$. Let $x_{1}, x_{2}, \ldots, x_{n}$ be a path in $V_{G}$ of length not more than $a(c+b)$ with $x_{1}=x$ and $x_{n}=(\psi \circ \phi)(x)$. So

$|f((\psi \circ \phi)(x))-f(x)|^{p}=\left|\sum_{k=1}^{n-1}\left(f\left(x_{k+1}\right)-f\left(x_{k}\right)\right)\right|^{p} \leq n^{p-1} \sum_{k=1}^{n-1}\left|f\left(x_{k+1}\right)-f\left(x_{k}\right)\right|^{p}$.

The last sum approaches zero as $d_{G}\left(o_{G}, x\right) \rightarrow \infty$ since $f \in D_{p}(G)$ and $n \leq a(c+b)$. Thus $\lim _{d_{G}\left(o_{G}, x\right) \rightarrow \infty}|f((\psi \circ \phi)(x))-f(x)|=0$.

Proposition 6.4. The function $\bar{\phi}$ is a bijection.

Proof. Let $x_{1}, x_{2} \in \partial_{p}(G)$ with $x_{1} \neq x_{2}$, and let $f \in \mathrm{BD}_{p}(G)$ with $f\left(x_{1}\right) \neq f\left(x_{2}\right)$. There exists sequences $\left(x_{n}\right)$ and $\left(x_{n}^{\prime}\right)$ in $V_{G}$ such that $\left(x_{n}\right) \rightarrow x_{1}$ and $\left(x_{n}^{\prime}\right) \rightarrow x_{2}$. Assume that

$$
\bar{\phi}\left(x_{1}\right)=\lim _{n \rightarrow \infty}\left(\phi\left(x_{n}\right)\right)=\lim _{n \rightarrow \infty}\left(\phi\left(x_{n}^{\prime}\right)\right)=\bar{\phi}\left(x_{2}\right),
$$

so $\lim _{n \rightarrow \infty} f\left((\psi \circ \phi)\left(x_{n}\right)\right)=\lim _{n \rightarrow \infty} f\left((\psi \circ \phi)\left(x_{n}^{\prime}\right)\right)$. It follows from Lemma 6.3 that $\lim _{n \rightarrow \infty} f\left(x_{n}\right)=\lim _{n \rightarrow \infty} f\left(x_{n}^{\prime}\right)$; thus $f\left(x_{1}\right)=f\left(x_{2}\right)$, a contradiction. Hence $\bar{\phi}$ is one-to-one.

We now show that $\bar{\phi}$ is onto. Let $y \in \partial_{p}(H)$ and let $\left(y_{n}\right)$ be a sequence in $V_{H}$ that converges to $y$. By passing to a subsequence if necessary, we can assume that there is a unique $x$ in the compact Hausdorff space $\operatorname{Sp}\left(\operatorname{BD}_{p}(G)\right)$ such that $\left(\psi\left(y_{n}\right)\right) \rightarrow x$. 
Since $\lim _{n \rightarrow \infty} d_{H}\left(o_{H}, y_{n}\right) \rightarrow \infty$, we have $\lim _{n \rightarrow \infty} d_{G}\left(o_{G}, \psi\left(y_{n}\right)\right) \rightarrow \infty$, so $x \notin G$. Using an argument similar to the first paragraph in the proof of Proposition 6.2, we obtain $x \in \partial_{p}(G)$. The proof will be complete once we show that $\bar{\phi}(x)=y$. Let $f \in \mathrm{BD}_{p}(H)$. By Lemma 6.3, we see that $\lim _{n \rightarrow \infty}\left|f\left((\phi \circ \psi)\left(y_{n}\right)\right)-f\left(y_{n}\right)\right|=0$. Thus $f(\bar{\phi}(x))=f(y)$ for all $f \in \mathrm{BD}_{p}(H)$. Hence $\bar{\phi}(x)=y$.

We finally show that the bijection $\bar{\phi}$ is also a homeomorphism. We only need to show that $\bar{\phi}$ is continuous, since both $\operatorname{Sp}\left(\mathrm{BD}_{p}(G)\right)$ and $\operatorname{Sp}\left(\mathrm{BD}_{p}(H)\right)$ are compact Hausdorff spaces. Let $W$ be an open set in $\partial_{p}(H)$ and let $x \in \bar{\phi}^{-1}(W)$. Choose $y \in W$ so that $x=\bar{\phi}^{-1}(y)$. By Proposition 4.12, there exists a subset $U$ of $V_{H}$ such that $y \in \bar{U}$ and $\bar{U} \cap \partial_{p}(H) \subseteq W$. We saw in the proof of Proposition 4.12 that there is an $h \in \operatorname{BHD}_{p}(H)$ for which $\hat{h}(y)=1$ and $\hat{h}=0$ on $\partial_{p}(H) \backslash W$ and $\hat{h} \geq \epsilon$ on $\bar{U}$, where $0<\epsilon<1$. By Lemma 6.1(a), we have $\phi^{*} h=h \circ \phi \in \mathrm{BD}_{p}(G)$. Combining Theorems 4.6 and 4.8, we have an $\bar{h} \in \operatorname{BHD}_{p}(G)$ that satisfies $\bar{h}=\hat{h} \circ \bar{\phi}$ on $\partial_{p}(G)$. Let $O=\left\{x^{\prime} \in \partial_{p}(G) \mid \bar{h}\left(x^{\prime}\right)>\epsilon\right\}$. Now $O$ is an open set containing $x$ since $\bar{h}$ is continuous on $\partial_{p}(G)$ and $\bar{h}(x)=1$. For $z \in O$, we see that $\hat{h}(\bar{\phi}(z))=\bar{h}(z) \geq \epsilon$, thus $\bar{\phi}(z) \in W$ for all $z$ in $O$. Thus $O \subseteq \bar{\phi}^{-1}(W)$. Since our choice of $x$ was arbitrary, $\bar{\phi}^{-1}(W)$ is open and consequently $\bar{\phi}$ is continuous. The proof that $\bar{\phi}$ is a homeomorphism is complete.

We now prove Theorem 2.9. Let $\phi$ be a rough isometry from $G$ to $H$, and let $\psi$ be a rough inverse of $\phi$. Let $h \in \mathrm{BHD}_{p}(G)$. By Lemma 6.1(a), $h \circ \psi \in \mathrm{BD}_{p}(H)$. Let $\pi(h \circ \psi)$ be the unique element in $\operatorname{BHD}_{p}(H)$ given by Theorem 4.6. We now define a map $\Phi: \operatorname{BHD}_{p}(G) \mapsto \operatorname{BHD}_{p}(H)$ by $\Phi(h)=\pi(h \circ \psi)$. Theorem 4.8 implies that $\pi(h \circ \psi)(\bar{\phi}(x))=(h \circ \psi)(\bar{\phi}(x))$ for all $x \in \partial_{p}(G)$, where $\bar{\phi}$ is the homeomorphism from $\partial_{p}(G)$ to $\partial_{p}(H)$ defined earlier in this section. Thus $\Phi(h)(\bar{\phi}(x))=(h \circ \psi)(\bar{\phi}(x))=h(x)$ for all $x \in \partial_{p}(G)$. We can now show that $\Phi$ is one-to-one. Let $h_{1}, h_{2} \in \operatorname{BHD}_{p}(G)$ and suppose that $\Phi\left(h_{1}\right)=\Phi\left(h_{2}\right)$. So $\Phi\left(h_{1}\right)(\bar{\phi}(x))=\Phi\left(h_{2}\right)(\bar{\phi}(x))$ for all $x \in \partial_{p}(G)$, which implies $h_{1}(x)=h_{2}(x)$ for all $x \in \partial_{p}(G)$. Hence, $h_{1}=h_{2}$ by Corollary 4.9. Thus $\Phi$ is one-to-one.

We will now show that $\Phi$ is onto. Let $f \in \operatorname{BHD}_{p}(H)$. Then $f \circ \phi \in \mathrm{BD}_{p}(G)$. Let $h=\pi(f \circ \phi)$, where $\pi(f \circ \phi)$ is the unique element in $\operatorname{BHD}_{p}(G)$ given by Theorem 4.6. Let $y \in \partial_{p}(H)$. Since $h(x)=\pi(f \circ \phi)(x)$ for all $x \in \partial_{p}(G)$ and $\psi \circ \bar{\phi}$ equals the identity on $\partial_{p}(G)$, we see that $(\Phi(h))(y)=\pi(h \circ \psi)(y)=h(\psi(y))=$ $f((\phi \circ \psi)(y))=f(y)$. Thus $\Phi$ is onto and the proof of Theorem 2.9 is complete.

The map $\Phi$ is an isomorphism in the case $p=2$ since $\mathrm{BHD}_{2}(G)$ and $\mathrm{BHD}_{2}(H)$ are linear spaces. However, in general these spaces are not linear if $p \neq 2$.

\section{The first reduced $\ell^{p}$-cohomology of $\Gamma$}

In the final two sections, $\Gamma$ will denote a finitely generated group with generating set $S$. So for a real-valued function $f$ on $\Gamma$ the $p$-th power of the gradient and the 
$p$-Laplacian of $x \in \Gamma$ are

$$
\begin{aligned}
|D f(x)|^{p} & =\sum_{s \in S}\left|f\left(x s^{-1}\right)-f(x)\right|^{p}, \\
\Delta_{p} f(x) & =\sum_{s \in S}\left|f\left(x s^{-1}\right)-f(x)\right|^{p-2}\left(f\left(x s^{-1}\right)-f(x)\right) .
\end{aligned}
$$

If $f \in D_{p}(\Gamma)$, then $\left(\|f\|_{D_{p}}=I_{p}(f, \Gamma)+|f(e)|^{p}\right)^{1 / p}$, where $e$ is the identity element of $\Gamma$. Also $\ell^{p}(\Gamma)$ is the set that consists of real-valued functions on $\Gamma$ for which $\sum_{x \in \Gamma}|f(x)|^{p}$ is finite. The first reduced $\ell^{p}$-cohomology space of $\Gamma$ is defined by

$$
\bar{H}_{(p)}^{1}(\Gamma)=D_{p}(\Gamma) /\left(\overline{\ell^{p}(\Gamma) \oplus \mathbb{R}}\right)_{D_{p}} .
$$

We now prove Theorem 2.10. Suppose $\partial_{p}(\Gamma)=\varnothing$. By Proposition 4.2, there exists a sequence $\left(f_{n}\right)$ in $\mathbb{R} \Gamma$ that satisfies $\left\|f_{n}-1_{\Gamma}\right\|_{D_{p}} \rightarrow 0$. It follows that $I_{p}\left(f_{n}, \Gamma\right) \rightarrow 0$ and $\left(f_{n}(e)\right) \not \rightarrow 0$. Thus $\bar{H}_{(p)}^{1}(\Gamma)=0$ by [Puls 2003, Theorem 3.2]. We now assume $\partial_{p}(G) \neq \varnothing$. It was shown in [Puls 2006, Theorem 3.5] that $\bar{H}_{(p)}^{1}(\Gamma) \neq 0$ if and only if $\operatorname{HD}_{p}(\Gamma) \neq \mathbb{R}$. Since $\#(S)<\infty$, [Holopainen and Soardi 1997a, Lemma 4.4] says that $\operatorname{BHD}_{p}(\Gamma)=\mathbb{R}$ if and only if $\operatorname{HD}_{p}(\Gamma)=\mathbb{R}$. Theorem 2.10 now follows from Theorem 4.10.

We now use Theorem 2.10 to compute $\partial_{p}(\Gamma)$ and $R_{p}(\Gamma)$ for some special cases of $\Gamma$. By [Holopainen and Soardi 1997b, Corollary 1.10], $\operatorname{BHD}_{p}(\Gamma)=\mathbb{R}$ when $\Gamma$ has polynomial growth and $1<p \in \mathbb{R}$. Thus, if $\Gamma$ has polynomial growth, then $\bar{H}_{(p)}^{1}(\Gamma)=0$ and $\partial_{p}(\Gamma)$ is either the empty set or contains exactly one element. It would be nice to know when a group with polynomial growth is $p$-parabolic or $p$-hyperbolic. This has been worked out for the case $\Gamma=\mathbb{Z}^{n}$, where $n$ is a positive integer. Yamasaki [1977, Example 4.1] showed that $\mathbb{Z}$ is $p$-parabolic for $p>1$, and thus $\partial_{p}(\mathbb{Z})=\varnothing$ for $p>1$. The main result of [Maeda 1977] says that $\mathbb{Z}^{n}$ with $n \geq 2$ is $p$-parabolic if and only if $p \geq n$. Hence, $\partial_{p}\left(\mathbb{Z}^{n}\right)=\varnothing$ if $p \geq n$ and $\partial_{p}\left(\mathbb{Z}^{n}\right)$ consists of exactly one point if $1<p<n$.

There is a one-to-one correspondence between the maximal ideals of $\operatorname{BD}_{p}(\Gamma)$ and the points of $\operatorname{Sp}\left(\operatorname{BD}_{p}(\Gamma)\right)$. If $\tau \in R_{p}(\Gamma)$, then $\operatorname{ker}(\tau)$ is the maximal ideal of $\mathrm{BD}_{p}(\Gamma)$ corresponding to $\tau$. For each $x \in \Gamma$, we have $\delta_{x} \in \operatorname{ker}(\tau)$. By the continuity of $\tau$, we see that $\ell^{p}(\Gamma) \subseteq \operatorname{ker}(\tau)$. Assume that $\Gamma$ is nonamenable. Then $\ell^{p}(\Gamma)$ is closed in $D_{p}(\Gamma)$ by [Guichardet 1977, Corollary 1]. Hence $(\overline{\mathbb{R} \Gamma})_{D_{p}}=\ell^{p}(\Gamma)$. Also, $\left(\overline{\ell^{p}(\Gamma)}\right)_{\mathrm{BD}_{p}}=\ell^{p}(\Gamma)$ because $\left(\overline{\ell^{p}(\Gamma)}\right)_{\mathrm{BD}_{p}} \subseteq B\left(\overline{\ell^{p}(\Gamma)}\right)_{D_{p}}$. Thus $\hat{f}(\tau)=0$ for every $f \in(\overline{\mathbb{R}} \Gamma)_{D_{p}}$. Therefore, $R_{p}(\Gamma)=\partial_{p}(\Gamma)$ when $\Gamma$ is nonamenable. Consequently, $R_{p}(\Gamma)$ contains exactly one point when $\Gamma$ is nonamenable and $\bar{H}_{(p)}^{1}(\Gamma)=0$. Some groups that satisfy this last condition for $1<p \in \mathbb{R}$ are nonamenable groups with infinite center [Martin and Valette 2007, Theorem 4.2], and $\Gamma_{1} \times \Gamma_{2} \times \cdots \times \Gamma_{n}$ for $n \geq 2$, each $\Gamma_{i}$ is finitely generated, and at least one of the $\Gamma_{i}$ is nonamenable [Martin and Valette 2007, Theorem 4.7]. 


\section{Translation invariant linear functionals}

Recall that $\Gamma$ denotes a finitely generated group with generating set $S$. In this section we will study TILFs on $D_{p}(\Gamma) / \mathbb{R}$. By definition we have the inclusions

$$
\operatorname{Diff}\left(\ell^{p}(\Gamma)\right) \subseteq \operatorname{Diff}\left(D_{p}(\Gamma) / \mathbb{R}\right) \subseteq \ell^{p}(\Gamma) \subseteq D_{p}(\Gamma) / \mathbb{R} .
$$

The set $D_{p}(\Gamma) / \mathbb{R}$ is a Banach space under the norm induced from $I_{p}(\cdot, \Gamma)$. Thus if $[f]$ if a class from $D_{p}(G) / \mathbb{R}$, then its norm is given by

$$
\|[f]\|_{D(p)}=\left(\sum_{x \in \Gamma} \sum_{s \in S}\left|f\left(x s^{-1}\right)-f(x)\right|^{p}\right)^{1 / p} .
$$

We will write $\|f\|_{D(p)}$ for $\|[f]\|_{D(p)}$. Now $\left(\overline{\ell^{p}(\Gamma)}\right)_{D(p)}=D_{p}(\Gamma) / \mathbb{R}$ if and only if $\left(\overline{\ell^{p}(\Gamma) \oplus \mathbb{R}}\right)_{D_{p}}=D_{p}(\Gamma)$. So $\bar{H}_{(p)}^{1}(\Gamma)=0$ if and only if $\left(\overline{\ell^{p}(\Gamma)}\right)_{D(p)}=D_{p}(\Gamma) / \mathbb{R}$.

Lemma 8.1. $\left(\overline{\operatorname{Diff}\left(D_{p}(\Gamma) / \mathbb{R}\right)}\right)_{D(p)}=\left(\overline{\ell^{p}(\Gamma)}\right)_{D(p)}$.

Proof. Let $f \in \ell^{p}(\Gamma)$. By [Woodward 1974, Lemma 1], there is a sequence $\left(f_{n}\right)$ in $\operatorname{Diff}\left(\ell^{p}(\Gamma)\right)$ that converges to $f$ in the $\ell^{p}$-norm. It follows from Minkowski's inequality that for $s \in S$,

$$
\left\|\left(f-f_{n}\right)_{s}-\left(f-f_{n}\right)\right\|_{p}^{p}=\sum_{x \in \Gamma}\left|f\left(x s^{-1}\right)-f_{n}\left(x s^{-1}\right)-\left(f(x)-f_{n}(x)\right)\right|^{p} \rightarrow 0
$$

as $n \rightarrow \infty$. Hence $f \in\left(\overline{\operatorname{Diff}\left(\ell^{p}(\Gamma)\right)}\right)_{D(p)}$, implying $\ell^{p}(\Gamma) \subseteq\left(\overline{\operatorname{Diff}\left(\ell^{p}(\Gamma)\right)}\right)_{D(p)}$. The result now follows.

Theorem 8.2. Let $1<p \in \mathbb{R}$. Then $\bar{H}_{(p)}^{1}(\Gamma) \neq 0$ if and only if there exists a nonzero continuous TILF on $D_{p}(\Gamma) / \mathbb{R}$.

Proof. If $\bar{H}_{(p)}^{1}(\Gamma) \neq 0$, then $\left(\overline{\ell^{p}(\Gamma)}\right)_{D(p)} \neq D_{p}(\Gamma) / \mathbb{R}$. It now follows from the Hahn-Banach theorem that there exists a nonzero continuous linear functional $T$ on $D_{p}(\Gamma) / \mathbb{R}$ such that $\left(\overline{\ell^{p}(\Gamma)}\right)_{D(p)}$ is contained in the kernel of $T$. Thus $T$ is translation invariant by Lemma 8.1 .

Conversely, if $T$ is a continuous TILF on $D_{p}(\Gamma) / \mathbb{R}$, then $T(f)=0$ for all $f \in\left(\overline{\ell^{p}(\Gamma)}\right)_{D(p)}$. So if there exists a nonzero continuous TILF on $D_{p}(\Gamma) / \mathbb{R}$, then $\left(\overline{\ell^{p}(\Gamma)}\right)_{D(p)} \neq D_{p}(\Gamma) / \mathbb{R}$.

Theorem 2.11 now follows by combining Theorems 8.2 and 2.10.

If $h \in D_{p}(\Gamma) / \mathbb{R}$, then $\left\langle\triangle_{p} h, \cdot\right\rangle$ is a well-defined continuous linear functional on $D_{p}(\Gamma) / \mathbb{R}$ since equivalent functions in $D_{p}(\Gamma) / \mathbb{R}$ differ by a constant. It was shown in [Puls 2006, Proposition 3.4] that if $h \in \operatorname{HD}_{p}(\Gamma) / \mathbb{R}$ and $f \in\left(\overline{\ell^{p}(\Gamma)}\right)_{D(p)}$, then $\left\langle\triangle_{p} h, f\right\rangle=0$. Consequently, if $h \in \operatorname{HD}_{p}(\Gamma) / \mathbb{R}$, then $\left\langle\triangle_{p} h, \cdot\right\rangle$ defines a continuous TILF on $D_{p}(\Gamma) / \mathbb{R}$. Thus there are no nonzero continuous TILFs on $D_{p}(\Gamma) / \mathbb{R}$ when $\operatorname{HD}_{p}(\Gamma)$ only contains the constant functions. 
If $\bar{H}_{(p)}^{1}(\Gamma)=0$, then $\left(\overline{\ell^{p}(\Gamma)}\right)_{D(p)}=D_{p}(\Gamma) / \mathbb{R}$. It is known that $\ell^{p}(\Gamma)$ is closed in $D_{p}(\Gamma) / \mathbb{R}$ if and only if $\Gamma$ is nonamenable, [Guichardet 1977, Corollary 1]. As was mentioned in Section 2, if $\Gamma$ is nonamenable, then zero is the only TILF on $\ell^{p}(\Gamma)$. Consequently zero is the only TILF on $D_{p}(\Gamma) / \mathbb{R}$ when $\Gamma$ is nonamenable and $\bar{H}_{(p)}^{1}(\Gamma)=0$. Summing up:

Theorem 8.3. Let $\Gamma$ be an infinite, finitely generated group and let $1<p \in \mathbb{R}$. The following are equivalent:

(1) $\bar{H}_{(p)}^{1}(\Gamma)=0$.

(2) Either $\partial_{p}(\Gamma)=\varnothing$ or $\#\left(\partial_{p}(\Gamma)\right)=1$.

(3) $\operatorname{HD}_{p}(\Gamma)=\mathbb{R}$.

(4) $\operatorname{BHD}_{p}(\Gamma)=\mathbb{R}$.

(5) The only continuous TILF on $D_{p}(\Gamma) / \mathbb{R}$ is zero. If $\Gamma$ is also nonamenable, then this is still equivalent to (6):

(6) Zero is the only TILF on $D_{p}(\Gamma) / \mathbb{R}$.

Some examples show zero is not the only TILF on $D_{p}(\Gamma) / \mathbb{R}$ when $\Gamma$ is nonamenable; this differs from the $\ell^{p}(\Gamma)$ case. Puls [2006, Corollary 4.3] showed $\bar{H}_{(p)}^{1}(\Gamma) \neq 0$ for groups with infinitely many ends and $1<p \in \mathbb{R}$. Thus by Theorem 8.2 there exists a nonzero continuous TILF on $D_{p}(\Gamma) / \mathbb{R}$.

If there is a nonzero continuous TILF on $D_{r}(\Gamma) / \mathbb{R}$ for some nonamenable group $\Gamma$ and some real number $r$, then is it true that there is a nonzero continuous TILF on $D_{p}(\Gamma) / \mathbb{R}$ for all real numbers $p>1$ ? The answer to this question is no. To see this, let $\mathscr{H}^{n}$ denote hyperbolic $n$-space, and suppose $\Gamma$ is a group that acts properly discontinuously on $\mathscr{H}^{n}$ by isometries and that the action is cocompact and free. By combining [Bourdon et al. 2005, Theorem 2] and [Puls 2007, Theorem 1.1], we obtain $\bar{H}_{(p)}^{1}(\Gamma) \neq 0$ if and only if $p>n-1$.

\section{Acknowledgments}

I would like to thank Peter Linnell for many useful comments on a preliminary version of this paper. I would also like to thank the referee for some suggestions that greatly improved the exposition of the paper.

\section{References}

[Bekka and Valette 1997] M. E. B. Bekka and A. Valette, "Group cohomology, harmonic functions and the first $L^{2}$-Betti number”, Potential Anal. 6 (1997), 313-326. MR 98e:20056 Zbl 0882.22013

[Bourdon 2004] M. Bourdon, "Cohomologie $l_{p}$ et produits amalgamés", Geometriae Dedicata 107 (2004), 85-98. MR 2005j:20047 Zbl 1124.20025 
[Bourdon et al. 2005] M. Bourdon, F. Martin, and A. Valette, "Vanishing and non-vanishing for the first $L^{p}$-cohomology of groups", Comment. Math. Helv. 80:2 (2005), 377-389. MR 2006a:20097 Zbl 1139.20045

[Cheeger and Gromov 1986] J. Cheeger and M. Gromov, " $L_{2}$-cohomology and group cohomology", Topology 25:2 (1986), 189-215. MR 87i:58161 Zbl 0597.57020

[Coulhon et al. 2001] T. Coulhon, I. Holopainen, and L. Saloff-Coste, "Harnack inequality and hyperbolicity for subelliptic p-Laplacians with applications to Picard type theorems", Geom. Funct. Anal. 11:6 (2001), 1139-1191. MR 2003g:31006a Zbl 1005.58013

[Ekeland and Témam 1999] I. Ekeland and R. Témam, Convex analysis and variational problems, Classics in Applied Mathematics 28, Society for Industrial and Applied Mathematics (SIAM), Philadelphia, PA, 1999. MR 2000j:49001 Zbl 0939.49002

[Elek 1997] G. Elek, "The $l_{p}$-cohomology and the conformal dimension of hyperbolic cones", $G e$ ometriae Dedicata 68:3 (1997), 263-279. MR 99b:53062 Zbl 0899.53035

[Elek 1998] G. Elek, "Coarse cohomology and $l_{p}$-cohomology", K-Theory 13:1 (1998), 1-22. MR 99a:58148 Zbl 0899.46059

[Gol'dshteĭn et al. 1987] V. M. Gol'dshteĭn, V. I. Kuz'minov, and I. A. Shvedov, “ $L_{p}$-cohomology of Riemannian manifolds", Trudy Inst. Mat. (Novosibirsk) 7 (1987), 101-116. In Russian. MR 89d: $58117 \mathrm{Zbl} 0654.58030$

[Grigoryan 1987] A. A. Grigoryan, "On Liouville theorems for harmonic functions with finite Dirichlet integral”, Mat. Sb. (N.S.) 132(174):4 (1987), 496-516, 592. In Russian; translated in Math. USSR Sb., 60:2 (1988), 485-504. MR 88g:35068 Zbl 0646.31009

[Gromov 1993] M. Gromov, "Asymptotic invariants of infinite groups", pp. 1-295 in Geometric group theory (Sussex, 1991), vol. 2, London Math. Soc. Lecture Note Ser. 182, Cambridge Univ. Press, Cambridge, 1993. MR 95m:20041 Zbl 0841.20039

[Guichardet 1977] A. Guichardet, "Étude de la $l$-cohomologie et de la topologie du dual pour les groupes de Lie à radical abélien”, Math. Ann. 228:3 (1977), 215-232. MR 56 \#539 Zbl 0337.22017

[Holopainen and Soardi 1997a] I. Holopainen and P. M. Soardi, " $p$-harmonic functions on graphs and manifolds”, Manuscripta Math. 94:1 (1997), 95-110. MR 99c:31017 Zbl 0898.31007

[Holopainen and Soardi 1997b] I. Holopainen and P. M. Soardi, "A strong Liouville theorem for $p$ harmonic functions on graphs", Ann. Acad. Sci. Fenn. Math. 22:1 (1997), 205-226. MR 97m:31004 Zbl 0874.31008

[Kim and Lee 2005] S. W. Kim and Y. H. Lee, "Positive p-harmonic functions on graphs", Bull. Korean Math. Soc. 42:2 (2005), 421-432. MR 2006a:31006 Zbl 1080.31006

[Kim and Lee 2007] S. W. Kim and Y. H. Lee, "Energy finite p-harmonic functions on graphs and rough isometries", Commun. Korean Math. Soc. 22:2 (2007), 277-287. MR 2008h:31013 Zbl 1168.31302

[Maeda 1977] F.-Y. Maeda, "A remark on parabolic index of infinite networks", Hiroshima Math. J. 7:1 (1977), 147-152. MR 55 \#2396 Zbl 0382.90089

[Martin and Valette 2007] F. Martin and A. Valette, "On the first $L^{p}$-cohomology of discrete groups", Groups Geom. Dyn. 1:1 (2007), 81-100. MR 2007m:20078 Zbl 1175.20045

[Meisters 1983] G. H. Meisters, "Some problems and results on translation-invariant linear forms", pp. 423-444 in Radical Banach algebras and automatic continuity (Long Beach, CA, 1981), Lecture Notes in Math. 975, Springer, Berlin, 1983. MR 85c:46035 Zbl 0507.46022

[Pansu 1989] P. Pansu, "Cohomologie $L^{p}$ des variétés à courbure négative, cas du degré 1", Rend. Sem. Mat. Univ. Politec. Torino Special issue (1989), 95-120. MR 92e:58200 Zbl 0723.53023 
[Pansu 2007] P. Pansu, "Cohomologie $L^{p}$ en degré 1 des espaces homogènes", Potential Anal. 27:2 (2007), 151-165. MR 2008h:43005 Zbl 05181216

[Pansu 2008] P. Pansu, "Cohomologie $L^{p}$ et pincement", Comment. Math. Helv. 83:2 (2008), 327357. MR 2009j:53034 Zbl 1142.53023

[Puls 2003] M. J. Puls, "Group cohomology and $L^{p}$-cohomology of finitely generated groups", Canad. Math. Bull. 46:2 (2003), 268-276. MR 2004e:43003 Zbl 1030.43001

[Puls 2006] M. J. Puls, "The first $L^{p}$-cohomology of some finitely generated groups and $p$-harmonic functions”, J. Funct. Anal. 237:2 (2006), 391-401. MR 2008e:43003 Zbl 1094.43003

[Puls 2007] M. J. Puls, "The first $L^{p}$-cohomology of some groups with one end", Arch. Math. (Basel) 88:6 (2007), 500-506. MR 2008m:43004 Zbl 1124.43002

[Saeki 1984] S. Saeki, "Discontinuous translation invariant functionals", Trans. Amer. Math. Soc. 282:1 (1984), 403-414. MR 85k:43004 Zbl 0537.43002

[Soardi 1993] P. M. Soardi, "Rough isometries and Dirichlet finite harmonic functions on graphs", Proc. Amer. Math. Soc. 119:4 (1993), 1239-1248. MR 94a:31004 Zbl 0801.31002

[Soardi 1994] P. M. Soardi, Potential theory on infinite networks, Lecture Notes in Mathematics 1590, Springer, Berlin, 1994. MR 96i:31005 Zbl 0818.31001

[Taylor and Lay 1986] A. E. Taylor and D. C. Lay, Introduction to functional analysis, 2nd ed., Robert E. Krieger, Melbourne, FL, 1986. MR 87k:46001 Zbl 0654.46002

[Tessera 2009] R. Tessera, "Vanishing of the first reduced cohomology with values in an $L^{p}$-representation", Ann. Inst. Fourier (Grenoble) 59:2 (2009), 851-876. MR 2010g:22036 Zbl 05549010

[Willis 1986] G. A. Willis, "Translation invariant functionals on $L^{p}(G)$ when $G$ is not amenable", J. Austral. Math. Soc. Ser. A 41:2 (1986), 237-250. MR 88b:43002 Zbl 0611.43001

[Willis 1988] G. A. Willis, "Continuity of translation invariant linear functionals on $C_{0}(G)$ for certain locally compact groups G", Monatsh. Math. 105:2 (1988), 161-164. MR 89c:43004 Zbl 0637. 43003

[Woodward 1974] G. S. Woodward, "Translation-invariant linear forms on $C_{0}(G), C(G), L^{p}(G)$ for noncompact groups", J. Functional Analysis 16 (1974), 205-220. MR 49 \#9540 Zbl 0278.43002

[Wysoczański 1996] J. Wysoczański, "Royden compactification of integers", Hiroshima Math. J. 26:3 (1996), 515-529. MR 97h:54027 Zbl 0881.54029

[Yamasaki 1977] M. Yamasaki, "Parabolic and hyperbolic infinite networks", Hiroshima Math. J. 7:1 (1977), 135-146. MR 55 \#2395 Zbl 0382.90088

Received June 18, 2008. Revised September 7, 2010.

Michael J. Puls

DEPARTMENT OF MATHEMATICS

JOHN JAY COLLEGE-CUNY

445 WEST 59TH STREET

NEW YORK, NY 10019

UNITED STATES

mpuls@jjay.cuny.edu 


\title{
PACIFIC JOURNAL OF MATHEMATICS
}

\author{
http://www.pjmath.org \\ Founded in 1951 by \\ E. F. Beckenbach (1906-1982) and F. Wolf (1904-1989)
}

\section{EDITORS}

V. S. Varadarajan (Managing Editor)

Department of Mathematics

University of California

Los Angeles, CA 90095-1555

pacific@math.ucla.edu

Vyjayanthi Chari

Department of Mathematics

University of California

Riverside, CA 92521-0135

chari@math.ucr.edu

Robert Finn

Department of Mathematics Stanford University

Stanford, CA 94305-2125

finn@math.stanford.edu

Kefeng Liu

Department of Mathematics

University of California

Los Angeles, CA 90095-1555

liu@math.ucla.edu
Darren Long

Department of Mathematics

University of California

Santa Barbara, CA 93106-3080

long@math.ucsb.edu

Jiang-Hua Lu

Department of Mathematics

The University of Hong Kong

Pokfulam Rd., Hong Kong jhlu@maths.hku.hk

Alexander Merkurjev

Department of Mathematics

University of California

Los Angeles, CA 90095-1555

merkurev@math.ucla.edu
Sorin Popa

Department of Mathematics University of California

Los Angeles, CA 90095-1555 popa@math.ucla.edu

Jie Qing

Department of Mathematics

University of California

Santa Cruz, CA 95064

qing@cats.ucsc.edu

Jonathan Rogawski

Department of Mathematics

University of California

Los Angeles, CA 90095-1555

jonr@math.ucla.edu

\section{PRODUCTION}

pacific@math.berkeley.edu

\begin{abstract}
Silvio Levy, Scientific Editor Matthew Cargo, Senior Production Editor
\end{abstract}
ACADEMIA SINICA, TAIPEI

CALIFORNIA INST. OF TECHNOLOGY

INST. DE MATEMÁTICA PURA E APLICADA

KEIO UNIVERSITY

MATH. SCIENCES RESEARCH INSTITUTE

NEW MEXICO STATE UNIV.

OREGON STATE UNIV.

\section{SUPPORTING INSTITUTIONS}

STANFORD UNIVERSITY
UNIV. OF BRITISH COLUMBIA
UNIV. OF CALIFORNIA, BERKELEY
UNIV. OF CALIFORNIA, DAVIS
UNIV. OF CALIFORNIA, LOS ANGELES
UNIV. OF CALIFORNIA, RIVERSIDE
UNIV. OF CALIFORNIA, SAN DIEGO
UNIV. OF CALIF., SANTA BARBARA

UNIV. OF CALIF., SANTA CRUZ

UNIV. OF MONTANA

UNIV. OF OREGON

UNIV. OF SOUTHERN CALIFORNIA

UNIV. OF UTAH

UNIV. OF WASHINGTON

WASHINGTON STATE UNIVERSITY

These supporting institutions contribute to the cost of publication of this Journal, but they are not owners or publishers and have no responsibility for its contents or policies.

See inside back cover or www.pjmath.org for submission instructions.

The subscription price for 2010 is US \$420/year for the electronic version, and \$485/year for print and electronic.

Subscriptions, requests for back issues from the last three years and changes of subscribers address should be sent to Pacific Journal of Mathematics, P.O. Box 4163, Berkeley, CA 94704-0163, U.S.A. Prior back issues are obtainable from Periodicals Service Company, 11 Main Street, Germantown, NY 12526-5635. The Pacific Journal of Mathematics is indexed by Mathematical Reviews, Zentralblatt MATH, PASCAL CNRS Index, Referativnyi Zhurnal, Current Mathematical Publications and the Science Citation Index.

The Pacific Journal of Mathematics (ISSN 0030-8730) at the University of California, c/o Department of Mathematics, 969 Evans Hall, Berkeley, CA 94720-3840, is published monthly except July and August. Periodical rate postage paid at Berkeley, CA 94704, and additional mailing offices. POSTMASTER: send address changes to Pacific Journal of Mathematics, P.O. Box 4163, Berkeley, CA 94704-0163.

PJM peer review and production are managed by EditFLOW ${ }^{\mathrm{TM}}$ from Mathematical Sciences Publishers.

PUBLISHED BY PACIFIC JOURNAL OF MATHEMATICS

at the University of California, Berkeley 94720-3840

A NON-PROFIT CORPORATION

Typeset in LATEX

Copyright $(\mathrm{C} 2010$ by Pacific Journal of Mathematics 


\section{PACIFIC JOURNAL OF MATHEMATICS}

Volume $248 \quad$ No. $2 \quad$ December 2010

Topological description of Riemannian foliations with dense leaves

Jesús A. ÁlVAREZ LóPEZ and Alberto CANDEL

The nonexistence of quasi-Einstein metrics

JEFFREY S. CASE

Twisted symmetric group actions

AKINARI HoSHI and MING-CHANG KANG

Optimal transportation and monotonic quantities on evolving manifolds

305

HONG HUANG

Hopf structures on the Hopf quiver $Q(\langle g\rangle, g)$

HuA-Lin HuANG, YU Ye and QING ZHaO

Minimal surfaces in $S^{3}$ foliated by circles

Nikolai KuteV and VelichKa Milousheva

Prealternative algebras and prealternative bialgebras

XIANG Ni and CHENGMING BAI

Some remarks about closed convex curves

Ke OU and Shengliang PAN

Orbit correspondences for real reductive dual pairs

SHU-YEN PAN

Graphs of bounded degree and the $p$-harmonic boundary

Michael J. Puls

Invariance of the BFV complex

FLORIAN SCHÄTZ

Some elliptic PDEs on Riemannian manifolds with boundary

YANNICK SIRE and ENRICO VALDINOCI

Representations of Lie superalgebras in prime characteristic, III 